\title{
Meaning-dependent ratings of imagery, age of acquisition, familiarity, and concreteness for 387 ambiguous words
}

\author{
K. J. GILHOOLY and R. H. LOGIE \\ University of Aberdeen, King's College, Aberdeen AB9 2UB, Scotland
}

\begin{abstract}
The problem of word ambiguity has generally been overlooked in compiling lists of words measured on various attributes. In this study, rating measures were obtained on the meanings of 387 words, the ambiguity of which had been established empirically. Imagery, age-ofacquisition, concreteness, and familiarity ratings are reported for each meaning, together with an index of meaning dominance. The results suggest that the most dominant meanings tend to be the most imageable, concrete, familiar, and earliest acquired. Generally satisfactory correlations with other norms were obtained.
\end{abstract}

Many studies of verbal learning, memory, and perception have drawn stimulus material from lists of words measured on various dimensions such as imagery, concreteness, familiarity, meaningfulness, and age of acquisition. Typical lists are those presented by Gilhooly and Hay (1977), Paivio, Yuille, and Madigan (1968), and Toglia and Battig (1978). However, an important issue that has apparently been overlooked in constructing such lists is the problem of multiple meanings. For example, Britton (1978) estimated that approximately $44 \%$ of words in the American Heritage Dictionary of the English Language (1969) are ambiguous.

Differences in degree of word ambiguity may produce differences in "encoding variability" effects and underlie certain recall and recognition memory phenomena (Morris, 1978; Reder, Anderson, \& Bjork, 1974). Also, facilitating effects of ambiguity have been reported in lexical decision making (Rubenstein, Garfield, \& Millikan, 1970; Rubenstein, Lewis, \& Rubenstein, 1971). So, it seems that degree of word ambiguity should be taken into account in a number of verbal tasks. Furthermore, it is reasonable to suppose that the nature of the ambiguity should also be considered.

For example, in a task in which imagery is important, a word that has two equally salient and equally imageable meanings may be processed in similar ways even by subjects who respond on the basis of different meanings, whereas a word that has two equally salient but unequally imageable meanings is likely to lead to marked variation in processing over different subjects. In the case of rating production, different subjects might

This research was carried out with the assistance of (United Kingdom) SSRC Grant HR 5957. Requests for reprints should be addressed to K. J. Gilhooly, Department of Psychology, University of Aberdeen, King's College, Aberdeen AB9 2UB, Scotland. rate ambiguous words in terms of different meanings, possibly leading to spurious average values that do not represent any one subject's judgment. This point has been made by Toglia and Battig (1978, p. 12), using the word "felt" as an example. Some subjects may rate "felt" as highly concrete on the basis of its meaning as a type of cloth, whereas others may rate it as low concrete on the basis of its being the past tense of "feel." On the other hand, it may be that most ambiguous words have but one common encoding that is generally used unless the context suggests otherwise. It would seem desirable, in any case, to know which meanings of ambiguous words are most commonly thought of and are thus likely to be most commonly used in processing isolated words in a range of tasks.

To this end, the salience of the alternative meanings of ambiguous words must be assessed. Many alternative meanings given in dictionaries will be unknown, or of very low salience, to most people. For example, the word "clerk" may be effectively unambiguous to most subjects, but according to Chambers 20th Century Dictionary (1972), five meanings are associated with this word. In a previous study (Gilhooly \& Logie, 1980), the degree of effective ambiguity of 1,944 words was assessed in a two-stage process. First, all of the words were rated for degree of ambiguity by 17 subjects. Second, the words that were rated as ambig. uous by three or more subjects in the first phase were presented to 40 subjects, who were asked to write down for each word the first meaning that occurred to them. These subject-reported meanings formed the basic data for the present study. The frequencies of the different meanings produced for each word were tabulated, and the separate meanings were then rated by independent groups of subjects for age of acquisition, imagery, concreteness, and familiarity. This paper reports the different meanings and their frequencies for each ambig. 
uous word, together with the average ratings given to each of the meanings on the four dimensions mentioned above.

\section{METHOD}

\section{Selection of Words and Meanings}

Gilhooly and Logie (1980) reported a study in which subjects, given a word previously rated as ambiguous, were required to write down the first meaning that came to mind upon seeing the word. By this means, a dominance or salience hierarchy of meanings for each of the words was determined from the number of subjects producing each meaning. Meanings given by $10 \%$ (i.e., four) or more subjects were taken as being reasonably salient, for the purposes of producing disambiguated ratings on those words. This procedure resulted in the distribution given in Table 1 .

It was considered unnecessary to include the three words having five meanings, since these would have added 15 extra items to the task but would have yielded only unreliable extra information on meanings at the fifth level of dominance. Thus only words with two, three, or four salient meanings were used in the rating task. A total of 387 words with a total of 905 meanings were finally used.

\section{Ratings Procedure}

For the disambiguated ratings of age of acquisition, imagery, concreteness, and familiarity, the following procedure was used.

First, each salient meaning was denoted by a word or short phrase as unambiguously as possible, based largely on definitions from Collins English Gem Dictionary (Foreman, 1963). The words, each with an associated meaning, were then printed in random order, 20 to a page, and alongside each word was a 7-point rating scale. The restriction was imposed that a given word should not appear more than once on any page.

The pages were shuffled and assembled into booklets such that each booklet contained the pages in a different random order. The booklets were administered in one session to subjects who were asked to rate each word according to the instructions appropriate to the attribute for their group. Subjects were asked to rate each word solely on the basis of the meaning given. If a subject was unclear as to the meaning intended, this was clarified by the experimenter.

The instructions for ratings of each attribute were variants of those used by Gilhooly and Logie (1980), modified for disambiguated ratings. Instructions for the imagery ratings provide an example.

Imagery rating instructions. "Word meanings differ in their capacity to arouse mental images of things or events. Some word meanings arouse a sensory experience, such as a mental picture or sound, very quickly and easily, whereas others may do so only with difficulty (i.e., after a long delay) or not at all. The purpose of this experiment is to rate a list of word meanings as to the ease or difficulty with which they arouse mental images. Any word meaning which, in your estimation, arouses a mental

Table 1

Distribution of Meanings Given by at Least $10 \%$ of Subjects (Gilhooly \& Logie, 1980)

\begin{tabular}{rrr}
\hline M & W & T \\
\hline 2 & 269 & 358 \\
3 & 105 & 315 \\
4 & 13 & 52 \\
5 & 3 & 15 \\
& 390 & 920 \\
\hline
\end{tabular}

Note-M = number of meanings given by four or more subjects; $\boldsymbol{W}=$ number of words having exactly this number of salient meanings; $T=$ total number of meanings. image (i.e., a mental picture or sound, or other sensory experience) very quickly and easily should be given a high imagery rating; any word meaning that arouses a mental image with difficulty or not at all should be given a low imagery rating.

"Words can be ambiguous, and for a given word you may think of a number of different meanings. We are interested in the ratings given to different meanings of the same word, and so we have given you the meaning for each word on which you are to base your judgment. For instance, think of the word 'BRAND.' This may have the meaning 'BRAND (mark on cattle)' or 'BRAND (category of goods).' You may find that 'BRAND (mark on cattle)' arouses an image relatively easily and would be rated high on imagery, whereas 'BRAND (category of goods)' arouses a mental image with difficulty, if at all, and would be rated low on imagery. A word may have meanings other than those given; however, you are to rate the word only on the meaning that is given.

"Your ratings will be made on a 7-point scale, where 1 is the low-imagery end of the scale and 7 is the high-imagery end of the scale. Make your ratings by putting a circle around the number from 1 to 7 that best indicates your judgement of the ease or difficulty with which the word arouses imagery. The meanings that arouse mental images most readily for you should be given a rating of 7 ; meanings that arouse images with the greatest difficulty or not at all should be rated 1 ; meanings that are intermediate in ease or difficulty of imagery, of course, should be rated appropriately between the two extremes. Feel free to use the entire range of numbers, from 1 to 7 ; at the same time, don't be concerned about how often you use a particular number, as long as it is your true judgement. Work fairly quickly, but do not be careless in your ratings.

"Below are four examples. Indicate the ease of getting an image by circling the appropriate number.

$\begin{array}{lccccccc} & \text { Low } & & & & & & \text { High } \\ \text { CABLE (thick wire) } & 1 & 2 & 3 & 4 & 5 & 6 & 7 \\ \text { MAJOR (important) } & 1 & 2 & 3 & 4 & 5 & 6 & 7 \\ \text { CABLE (telegram) } & 1 & 2 & 3 & 4 & 5 & 6 & 7 \\ \text { MAJOR (rank in army) } & 1 & 2 & 3 & 4 & 5 & 6 & 7\end{array}$

"If necessary, refer back to these instructions when rating the word on the following pages. If there are any questions, ask them now. Otherwise, turn the page and begin."

The instructions for the other dimensions were similar, with changes appropriate to each dimension.

\section{Subjects}

The subjects were student volunteers attending Aberdeen University who were paid $£ 1.50$ for taking part. The distribution of subjects by sex and rating conditions was as follows: age of acquisition, 36 subjects (18 female, 18 male); imagery, 37 subjects ( 27 female, 10 male); familiarity, 35 subjects ( 23 female, 12 male); concreteness, 36 subjects ( 24 female, 12 male).

\section{RESULTS AND DISCUSSION}

Summary statistics on the four dimensions, organized by meaning-dependence level, are given in Table 2 . Data on the individual word meanings are given in the appendix. It appears from Table 2 that the most dominant meanings tend to be rated the most imageable, concrete, familiar, and earliest acquired. The reliabilities of the ratings at each dominance level were assessed by dividing the subjects into two groups, balanced as far as possible on sex and size, and calculating intergroup correlations. The resulting reliability coefficients (Table 2) were all satisfactory. In particular, the age ratings proved very reliable over the four dominance levels. 
Table 2

Ratings of Word Meanings: Summary Statistics Organized by Meaning-Dominance Level

\begin{tabular}{|c|c|c|c|c|}
\hline & I & AOA & F & $\mathrm{C}$ \\
\hline & \multicolumn{4}{|c|}{ Dominance Level $1(n=387)$} \\
\hline Mean & 4.81 & 4.10 & 5.06 & 4.87 \\
\hline SD & .96 & 1.02 & .74 & .87 \\
\hline Reliability & .88 & .96 & .82 & .86 \\
\hline \multirow[t]{2}{*}{${ }^{r_{A}}$} & .83 & .88 & .76 & .83 \\
\hline & \multicolumn{4}{|c|}{ Dominance Level $2(n=387)$} \\
\hline Mean & 4.43 & 4.50 & 4.40 & 4.38 \\
\hline SD & .94 & .97 & .81 & .82 \\
\hline Reliability & .83 & .95 & .82 & .83 \\
\hline \multirow[t]{2}{*}{${ }^{I_{A}}$} & .45 & .63 & .49 & .41 \\
\hline & \multicolumn{4}{|c|}{ Dominance Level $3(n=118)$} \\
\hline Mean & 4.22 & 4.67 & 4.20 & 4.19 \\
\hline SD & .91 & .99 & .77 & .80 \\
\hline Reliability & .80 & .95 & .80 & .84 \\
\hline \multirow[t]{2}{*}{$\mathbf{I}_{\mathbf{A}}$} & .65 & .64 & .54 & .63 \\
\hline & \multicolumn{4}{|c|}{ Dominance Level $4(n=13)$} \\
\hline Mean & 4.17 & 5.08 & 3.93 & 4.17 \\
\hline SD & .85 & .90 & 1.11 & .80 \\
\hline Reliability & .83 & .93 & .92 & .82 \\
\hline $\mathbf{I}_{\mathbf{A}}$ & .41 & .61 & .71 & .28 \\
\hline
\end{tabular}

Note-I = imagery, $A O A=$ age of acquisition, $F=$ familiarity, $C=$ concreteness; $r_{A}=$ correlation with ambiguous ratings.

For each word involved in the disambiguated rating procedure, measures were available from "normal" ambiguous ratings in which the word was simply presented with no meaning cue (Gilhooly \& Logie, 1980). As would be expected, the ratings of the more dominant meanings tended to correlate best with the ratings obtained from ambiguous presentation. This result is consistent with the view that ambiguous words are rated in terms of their most dominant meaning.

Some previous studies have reported results overlapping those noted in this paper, and comparisons were made. Overall, the correlations with previous studies were encouraging.

Cramer (1970) reported percentages of subjects giving each of two meanings to all members of a list of 100 ambiguous words. There was an overlap of 28 words and 53 meanings with the present study. The Pearson correlation between Cramer's percentage scores and our meaning-frequency scores was .81 over the 53 meanings. More recently, Nelson, McEvoy, Walling, and Wheeler (1980) reported the relative dominance of different meanings to 320 ambiguous words. There was an overlap of 70 words and 154 meanings between the Nelson et al. sample and the present study. The Pearson correlation between the Nelson et al. dominance measure and our meaning-frequency scores was .72 over the 154 shared meanings. Another relevant recent paper is the report by Wollen, Cox, Coahran, Shea, and Kirby (1980) of the relative frequencies of different meanings to 120 ambiguous words, together with concreteness ratings of each meaning. In this case, there was an overlap of 31 words and 63 meanings with the present study. The Pearson correlation between the Wollen et al. norms and our meaning-frequency scores was .34 . This is considerably lower than our correlations with Cramer and with Nelson et al. It is possible that, by accident of sampling, the particular words in common between the Wollen et al. study and the present study were more culture specific in their meaning-dominance patterns than average. Examining the 16 words and 33 meanings in common to Nelson et al., Wollen et al. and the present study, it was found that the Wollen et al. norms correlate only .49 with the Nelson et al. norms over those 33 meanings. This is markedly less than the correlation of .79 between the Nelson et al. and the Wollen et al. norms over the 148 meanings they have in common. Thus it is plausible that the sample of words common to Wollen et al. and the present paper are unusual and produce discrepancies with Nelson et al., as well as with our data. More encouragingly, a satisfactory degree of agreement was obtained between the Wollen et al. concreteness ratings and the present concreteness ratings ( $r=.84$ over 63 meanings). So, although there was some lack of agreement with Wollen et al. on meaning dominance, there was good agreement on how concrete the alternative meanings are. The present norms appear to exhibit reasonable consistency with those reported in previous studies.

\section{REFERENCES}

The American Heritage Dictionary of the English Language. Boston, Mass: Houghton Mifflin, 1969.

BritTon, B. K. Lexical ambiguity of words used in English text Behavior Research Methods \& Instrumentation, 1978, 10, 1-7. Chambers 20th Century Dictionary. London: Chambers, 1972.

Cramer, P. A study of homographs. In L. Postman \& G. Keppel (Eds.), Norms of word association. New York: Academic Press, 1970.

Foreman, J. B. (Ev.). Collins English gem dictionary. London: Collins, 1963.

Gilhooly, K. J., \& Hay, D. Imagery, concreteness, age-ofacquisition, familiarity, and meaningfulness values for 205 fiveletter words having single solution anagrams. Behavior Research Methods \& Instrumentation, 1977, 9, 12-17.

GilhooLy, K. J., \& LoGIE, R. H. Age-of-acquisition, imagery, concreteness, familiarity, and ambiguity measures for 1,944 words. Behavior Research Methods \& Instrumentation, 1980 12, 395-427.

Morris, P. E. Frequency and imagery in word recognition: Further evidence for an attribute model. British Journal of Psychology, 1978, 69, 69-75.

Nelson, D. L., McEvoy, C. L., Walling, J. R., \& Wheeler, J. W. The University of South Florida homograph norms. Behavior Research Methods \& Instrumentation, 1980, 12, 16-37.

Paivio, A., Yuille, J. D., \& Madigan, S. A. Concreteness, imagery and meaningfulness values for 925 nouns. Journal of Experimental Psychology: Monograph Supplement, 1968, 76(1, Pt. 2), 1-25. 
Reder, L. M., Anderson, J. R., \& BJork, R. A. A semantic interpretation of encoding specificity. Journal of Experimental Psychology, 1974, 102, 648-656.

Rubenstein, H., Garfiei.d, L., \& Millikan, J. A. Homographic entries in the internal lexicon. Journal of Verbal Learning and Verbal Behavior, 1970, 9, 487-494.

Rubenstein, H., Lewis, S. S., \& Rubenstein, M. A. Homographic entries in the internal lexicon: Effects of systematicity and relative frequency of meanings. Journal of Verbal Learning and Verbal Behavior, 1971, 10, 57-62.

Togilia, M. P., \& Battig, W. F. Handbook of semantic word norms. Hillsdale, N.J: Erlbaum, 1978.

Wollen, K. A., Cox, S. D., Coahran, M. M., Shea, D. S., \& $\mathrm{K}_{\mathrm{IRHY}}$, R. F. Frequency of occurrence and concreteness ratings of homograph meanings. Behavior Research Methods \& Instrumentation, 1980, 12, 8-15.

Appendix

Alphabetical Listing of the 387 Words and Each Meaning on Which Ratings Were Obtained

\begin{tabular}{|c|c|c|c|c|c|c|c|c|}
\hline \multirow[b]{2}{*}{ Word and Meaning } & \multicolumn{2}{|c|}{ Imagery } & \multicolumn{2}{|c|}{ Age of Acquisition } & \multicolumn{2}{|c|}{ Familiarity } & \multicolumn{2}{|c|}{ Concreteness } \\
\hline & Mean & SD & Mean & $\mathrm{SD}$ & Mean & $\mathrm{SD}$ & Mean & SD \\
\hline \multicolumn{9}{|l|}{$\mathrm{ACT}$} \\
\hline (19) pretence/performance & 4.49 & 1.80 & 4.36 & 1.00 & 5.06 & 1.35 & 4.53 & 1.74 \\
\hline $\begin{array}{l}\text { (15) part of play } \\
\text { ADDITION }\end{array}$ & 4.35 & 1.61 & 4.51 & .94 & 4.80 & 1.26 & 4.64 & 1.60 \\
\hline (26) arithmetic operation & 4.75 & 1.77 & 3.03 & .69 & 5.74 & 1.16 & 4.89 & 1.91 \\
\hline (10) extra & 2.87 & 1.49 & 4.60 & 1.05 & 4.91 & 1.20 & 3.25 & 1.89 \\
\hline \multicolumn{9}{|l|}{ AERIAL } \\
\hline (23) antenna & 5.46 & 1.59 & 4.41 & 1.29 & 4.63 & 1.85 & 5.67 & 1.67 \\
\hline (12) from the air & 4.05 & 1.72 & 5.58 & 1.16 & 4.20 & 1.58 & 3.72 & 2.00 \\
\hline \multicolumn{8}{|l|}{ AIM } & 2.09 \\
\hline (25) goal & 3.73 & 1.98 & 4.64 & 1.00 & 5.23 & 1.27 & 3.58 & 2.03 \\
\hline $\begin{array}{l}\text { (15) gauge direction of missile } \\
\text { ANSWER }\end{array}$ & 4.81 & 1.54 & 3.72 & .96 & 4.14 & 1.76 & 4.25 & 1.66 \\
\hline (30) response & 3.94 & 1.96 & 2.94 & .94 & 6.31 & 1.04 & 4.47 & 2.02 \\
\hline \multicolumn{8}{|l|}{ APPEAL } & 2.17 \\
\hline (28) plea & 4.49 & 1.84 & 5.08 & 1.01 & 4.89 & 1.43 & 3.83 & 1.76 \\
\hline \multicolumn{8}{|l|}{ APPROACH } & 1.57 \\
\hline (33) move towards & 4.22 & 1.77 & 4.11 & 1.17 & 5.27 & 1.27 & 4.63 & 1.64 \\
\hline $\begin{array}{l}\text { (06) way of thinking/acting } \\
\text { ARM }\end{array}$ & 2.95 & 1.54 & 5.42 & 1.04 & 4.60 & 1.57 & 2.86 & 1.57 \\
\hline (36) part of body & 6.42 & .89 & 1.94 & .74 & 6.66 & .63 & 6.47 & .96 \\
\hline $\begin{array}{l}\text { (04) to give a weapon } \\
\text { ARTICLE }\end{array}$ & 4.62 & 1.68 & 4.31 & .89 & 3.57 & 1.25 & 3.53 & 1.59 \\
\hline (29) object & 3.86 & 1.96 & 4.11 & .99 & 5.23 & 1.33 & 5.17 & 1.68 \\
\hline \multicolumn{8}{|l|}{ ASH } & 1.66 \\
\hline (29) solid remains of burning & 6.05 & 1.21 & 3.29 & 1.06 & 5.06 & 1.55 & 5.83 & 1.46 \\
\hline \multicolumn{8}{|l|}{ ATMOSPHERE } & 1.85 \\
\hline (33) gas surrounding heavenly body & 3.81 & 1.83 & 5.49 & 1.08 & 4.49 & 1.52 & 4.08 & 1.80 \\
\hline \multicolumn{9}{|l|}{ BACK } \\
\hline (35) hinder part of body/object & 4.83 & 2.22 & 2.97 & 1.42 & 5.71 & 1.47 & 5.50 & 1.76 \\
\hline \multicolumn{9}{|l|}{ BAG } \\
\hline (36) container & 5.92 & 1.38 & 2.39 & .79 & 6.17 & .81 & 6.22 & 1.11 \\
\hline $\begin{array}{l}\text { (04) unlikeable woman } \\
\text { BALANCE }\end{array}$ & 4.97 & 1.79 & 4.89 & 1.20 & 3.69 & 1.77 & 4.42 & 2.09 \\
\hline (17) equilibrium & 4.00 & 1.92 & 4.86 & 1.57 & 4.91 & 1.50 & 3.78 & 1.87 \\
\hline (15) scales for weighing & 5.05 & 1.45 & 4.06 & 1.07 & 4.43 & 1.32 & 5.00 & 1.76 \\
\hline \multicolumn{9}{|l|}{ BALL } \\
\hline (35) sphere/toy & 5.89 & 1.51 & 1.75 & .76 & 5.94 & 1.22 & 6.22 & 1.20 \\
\hline $\begin{array}{l}\text { (05) dance } \\
\text { BASE }\end{array}$ & 5.54 & 1.50 & 3.56 & 1.21 & 3.60 & 1.48 & 5.17 & 1.89 \\
\hline (24) bottom surface & 4.22 & 1.93 & 4.03 & 1.13 & 5.29 & 1.36 & 4.67 & 1.86 \\
\hline \multicolumn{9}{|l|}{ BATH } \\
\hline (35) cleaning receptacle & 6.22 & 1.23 & 2.28 & 1.12 & 6.29 & 88 & 6.36 & 1.27 \\
\hline (05) town in England & 4.32 & 1.88 & 5.17 & .99 & 3.46 & 1.84 & 4.69 & 2.04 \\
\hline
\end{tabular}




\begin{tabular}{|c|c|c|c|c|c|c|c|c|}
\hline \multirow[b]{2}{*}{ Word and Meaning } & \multicolumn{2}{|c|}{ Imagery } & \multicolumn{2}{|c|}{ Age of Acquisition } & \multicolumn{2}{|c|}{ Familiarity } & \multicolumn{2}{|c|}{ Concreteness } \\
\hline & Mean & SD & Mean & SD & Mean & SD & Mean & $\mathrm{SD}$ \\
\hline \multicolumn{9}{|l|}{ BEAM } \\
\hline (19) ray of light & 5.68 & 1.14 & 4.06 & 1.29 & 5.14 & 1.27 & 5.31 & 1.65 \\
\hline (17) support & 4.92 & 1.36 & 4.56 & 1.17 & 3.91 & 1.42 & 5.14 & 1.75 \\
\hline \multicolumn{9}{|l|}{ BEING } \\
\hline (22) type of creature & 3.65 & 1.98 & 4.54 & 1.20 & 4.77 & 1.55 & 4.06 & 1.90 \\
\hline (17) existing & 2.92 & 1.79 & 4.43 & 1.36 & 5.17 & 1.32 & 3.22 & 2.03 \\
\hline \multicolumn{9}{|l|}{ BID } \\
\hline (27) offer price & 4.22 & 1.78 & 5.11 & 1.07 & 4.83 & 1.46 & 3.69 & 2.04 \\
\hline (10) try for & 3.97 & 1.60 & 5.11 & 1.13 & 4.80 & 1.69 & 3.64 & 1.67 \\
\hline \multicolumn{9}{|l|}{ BITTERNESS } \\
\hline (25) sour taste & 4.49 & 2.00 & 3.97 & 1.21 & 5.03 & 1.38 & 5.08 & 1.69 \\
\hline (15) nasty/anger & 4.14 & 1.92 & 4.54 & 1.10 & 4.91 & 1.48 & 3.56 & 1.89 \\
\hline \multicolumn{9}{|l|}{ BLADE } \\
\hline (36) sharp piece of metal & 5.83 & 1.19 & 3.64 & 1.27 & 5.62 & 1.19 & 6.29 & .88 \\
\hline (04) piece of grass & 5.62 & 1.32 & 3.50 & 1.12 & 4.49 & 1.50 & 5.50 & 1.61 \\
\hline \multicolumn{9}{|l|}{ BLOCK } \\
\hline (15) impediment & 3.24 & 1.70 & 4.72 & 1.28 & 3.86 & 1.38 & 3.89 & 1.65 \\
\hline (15) solid piece of wood/stone & 5.35 & 1.73 & 3.20 & 1.19 & 5.26 & 1.32 & 6.00 & 1.31 \\
\hline (09) group of houses & 4.78 & 1.49 & 4.34 & 1.04 & 4.23 & 1.81 & 4.94 & 1.94 \\
\hline BODY & & & & & & & & \\
\hline (34) physical being & 5.17 & 1.80 & 3.56 & 1.52 & 5.49 & 1.32 & 6.11 & 1.17 \\
\hline (04) organisation & 2.95 & 1.33 & 5.42 & .92 & 3.74 & 1.38 & 3.53 & 1.69 \\
\hline BOLT & & & & & & & & \\
\hline (20) lock on door & 6.06 & 1.22 & 3.47 & .93 & 4.89 & 1.39 & 5.83 & 1.50 \\
\hline (12) run away & 4.30 & 1.72 & 4.34 & 1.07 & 3.77 & 1.27 & 4.00 & 1.89 \\
\hline (07) type of screw & 5.35 & 1.46 & 4.03 & .97 & 4.86 & 1.36 & 5.64 & 1.72 \\
\hline BOND & & & & & & & & \\
\hline (32) link & 4.38 & 1.88 & 5.17 & .93 & 4.74 & 1.44 & 3.92 & 1.83 \\
\hline (05) name & 4.24 & 2.26 & 4.83 & 1.30 & 2.34 & 1.55 & 3.19 & 2.25 \\
\hline BORDER & & & & & & & & \\
\hline (27) boundary between areas/countries & 4.89 & 1.74 & 4.11 & 1.20 & 5.63 & 1.42 & 4.36 & 2.07 \\
\hline (13) edge & 4.75 & 1.64 & 4.19 & 1.15 & 4.86 & 1.25 & 4.89 & 1.70 \\
\hline BOTHER & & & & & & & & \\
\hline (21) annoyance & 3.76 & 1.84 & 4.14 & 1.18 & 5.03 & 1.36 & 3.56 & 2.10 \\
\hline (09) violence/trouble & 4.05 & 1.61 & 4.74 & 1.23 & 4.49 & 1.54 & 3.31 & 1.61 \\
\hline (06) concerned about & 3.78 & 1.82 & 3.97 & 1.21 & 5.29 & 1.13 & 3.72 & 1.90 \\
\hline BOW & & & & & & & & \\
\hline (15) bend body & 4.24 & 1.73 & 3.89 & 1.35 & 3.46 & 1.44 & 3.75 & 1.71 \\
\hline (09) ribbon & 5.54 & 1.33 & 2.83 & 1.04 & 4.18 & 1.74 & 5.36 & 1.87 \\
\hline (07) weapon with arrows & 5.95 & 1.27 & 3.06 & .95 & 4.51 & 1.65 & 5.83 & 1.19 \\
\hline (06) musical device & 5.20 & 1.64 & 4.63 & 1.22 & 3.66 & 1.35 & 4.56 & 2.17 \\
\hline BOWL & & & & & & & & \\
\hline (33) container & 5.78 & 1.58 & 2.74 & .81 & 5.89 & 1.33 & 6.00 & 1.35 \\
\hline (06) throw a ball & 5.03 & 1.40 & 4.25 & 1.26 & 4.17 & 1.61 & 4.25 & 1.77 \\
\hline BOX & & & & & & & & \\
\hline (36) container & 5.94 & 1.41 & 2.39 & .86 & 6.06 & 1.15 & 6.19 & 1.33 \\
\hline (04) fight/sport & 4.92 & 1.53 & 3.97 & 1.07 & 4.20 & 1.31 & 4.28 & 1.57 \\
\hline BRASS & & & & & & & & \\
\hline (34) metal & 5.25 & 1.61 & 4.00 & 1.00 & 5.23 & 1.59 & 6.03 & 1.40 \\
\hline (04) musical group & 4.75 & 1.62 & 4.75 & 1.30 & 3.54 & 1.34 & 4.14 & 2.06 \\
\hline BRISTLE & & & & & & & & \\
\hline (25) part of brush & 5.51 & 1.50 & 3.92 & 1.23 & 5.14 & 1.42 & 5.72 & 1.66 \\
\hline (09) hair & 5.49 & 1.24 & 4.44 & 1.30 & 4.34 & 1.67 & 5.53 & 1.54 \\
\hline (04) sharp point & 5.42 & 1.48 & 4.47 & 1.42 & 3.32 & 1.39 & 5.23 & 1.68 \\
\hline BRUSH & & & & & & & & \\
\hline (22) cleaning equipment & 5.65 & 1.42 & 2.74 & 1.20 & 5.57 & 1.18 & 5.67 & 1.56 \\
\hline (08) for tidying hair & 6.43 & 1.08 & 2.56 & .76 & 6.00 & 1.10 & 6.00 & 1.29 \\
\hline$(05)$ for painting & 6.00 & 1.21 & 2.58 & .98 & 5.09 & 1.48 & 5.31 & 1.85 \\
\hline BUFFER & & & & & & & & \\
\hline (22) stop movement of train & 5.14 & 1.77 & 4.72 & 1.47 & 3.69 & 1.43 & 5.61 & 1.64 \\
\hline (14) chemical & 2.41 & 1.67 & 6.92 & .28 & 3.17 & 1.91 & 3.50 & 2.01 \\
\hline BUREAU & & & & & & & & \\
\hline (19) office & 5.00 & 1.87 & 5.29 & 1.00 & 4.63 & 1.42 & 5.47 & 1.55 \\
\hline (18) desk & 5.46 & 1.64 & 4.64 & 1.48 & 4.14 & 1.64 & 5.39 & 1.78 \\
\hline BURN & & & & & & & & \\
\hline (25) set on fire & 5.84 & 1.41 & 2.83 & .85 & 5.91 & 1.05 & 5.19 & 1.52 \\
\hline
\end{tabular}




\begin{tabular}{|c|c|c|c|c|c|c|c|c|}
\hline \multirow[b]{2}{*}{ Word and Meaning } & \multicolumn{2}{|c|}{ Imagery } & \multicolumn{2}{|c|}{ Age of Acquisition } & \multicolumn{2}{|c|}{ Familiarity } & \multicolumn{2}{|c|}{ Concreteness } \\
\hline & Mean & SD & Mean & SD & Mean & SD & Mean & SD \\
\hline (10) injury & 5.32 & 1.51 & 3.25 & 1.04 & 4.86 & 1.38 & 5.08 & 1.83 \\
\hline $\begin{array}{l}\text { (05) stream } \\
\text { BUSINESS }\end{array}$ & 5.14 & 1.76 & 3.89 & 1.76 & 4.23 & 1.77 & 4.94 & 2.03 \\
\hline (22) money-making concern & 4.16 & 1.87 & 4.64 & 1.03 & 5.40 & 1.42 & 4.42 & 1.83 \\
\hline (11) occupation & 3.84 & 1.78 & 4.77 & 1.22 & 4.60 & 1.36 & 3.92 & 1.59 \\
\hline $\begin{array}{l}\text { (06) affair/concern } \\
\text { CABINET }\end{array}$ & 3.57 & 1.82 & 4.33 & 1.43 & 4.94 & 1.24 & 3.56 & 1.86 \\
\hline (29) piece of furniture & 5.57 & 1.44 & 3.89 & 1.24 & 4.86 & 1.40 & 5.42 & 1.59 \\
\hline $\begin{array}{l}\text { (11) government body } \\
\text { CAPITAL }\end{array}$ & 4.24 & 1.53 & 5.78 & .92 & 4.57 & 1.57 & 4.47 & 2.13 \\
\hline (32) main town & 5.08 & 1.71 & 3.64 & 1.00 & 5.69 & 1.26 & 4.92 & 1.77 \\
\hline $\begin{array}{l}\text { (06) money } \\
\text { CARRIAGE }\end{array}$ & 4.03 & 1.91 & 5.61 & 1.01 & 3.97 & 1.75 & 3.64 & 1.72 \\
\hline (29) vehicle & 5.68 & 1.32 & 3.44 & .98 & 4.06 & 1.88 & 5.81 & 1.33 \\
\hline $\begin{array}{l}\text { (10) coach of train } \\
\text { CART }\end{array}$ & 5.97 & 1.15 & 3.72 & 1.10 & 5.14 & 1.05 & 5.75 & 1.46 \\
\hline (35) vehicle & 5.75 & 1.40 & 2.97 & .90 & 4.63 & 1.77 & 5.97 & 1.26 \\
\hline $\begin{array}{l}\text { (05) to carry } \\
\text { CASE }\end{array}$ & 4.61 & 1.74 & 3.89 & 1.24 & 4.43 & 1.27 & 4.00 & 1.76 \\
\hline (33) box/container & 5.27 & 1.41 & 3.44 & .93 & 5.06 & 1.22 & 5.72 & 1.61 \\
\hline $\begin{array}{l}\text { (05) law suit } \\
\text { CASTOR }\end{array}$ & 3.60 & 1.60 & 5.53 & .87 & 3.69 & 1.37 & 3.81 & 1.85 \\
\hline (20) small wheel & 4.32 & 1.90 & 4.78 & 1.40 & 4.03 & 1.50 & 5.28 & 2.05 \\
\hline (12) plant/oil & 3.53 & 2.14 & 4.63 & 1.29 & 3.82 & 1.69 & 4.56 & 2.11 \\
\hline $\begin{array}{l}\text { (07) type of sugar } \\
\text { CHAIN }\end{array}$ & 5.57 & 1.52 & 4.37 & 1.27 & 4.89 & 1.39 & 5.31 & 1.71 \\
\hline (34) metal links & 5.46 & 1.70 & 3.29 & 1.14 & 5.63 & 1.38 & 5.89 & 1.26 \\
\hline $\begin{array}{l}\text { (04) series } \\
\text { CHALLENGE }\end{array}$ & 3.22 & 1.62 & 4.64 & .89 & 4.14 & 1.42 & 3.33 & 1.63 \\
\hline (22) oppose/duel & 4.00 & 1.45 & 4.69 & 1.00 & 4.40 & 1.40 & 3.64 & 1.97 \\
\hline (11) difficult pursuit & 3.92 & 2.01 & 5.14 & 1.21 & 4.88 & 1.35 & 3.61 & 1.72 \\
\hline $\begin{array}{l}\text { (06) bet/dare } \\
\text { CHARACTER }\end{array}$ & 4.19 & 1.88 & 4.39 & .89 & 4.74 & 1.31 & 4.11 & 1.80 \\
\hline (28) type of person & 4.43 & 1.99 & 5.09 & 1.20 & 5.54 & 1.13 & 3.97 & 2.14 \\
\hline $\begin{array}{l}\text { (10) person in play/cartoon } \\
\text { CHARITY }\end{array}$ & 4.68 & 1.77 & 4.60 & 1.10 & 5.03 & 1.38 & 4.61 & 1.64 \\
\hline (26) giving & 3.75 & 1.92 & 4.43 & 1.15 & 4.71 & 1.56 & 3.14 & 2.00 \\
\hline $\begin{array}{l}\text { (14) organisation } \\
\text { CHARM }\end{array}$ & 4.39 & 1.75 & 4.34 & 1.07 & 5.11 & 1.47 & 4.50 & 1.72 \\
\hline (15) aspect of personality & 5.14 & 1.58 & 4.80 & 1.35 & 5.23 & 1.35 & 4.00 & 2.00 \\
\hline (11) bracelet/ring, etc. & 4.65 & 1.98 & 4.03 & 1.30 & 4.03 & 1.54 & 5.11 & 1.87 \\
\hline $\begin{array}{l}\text { (09) attraction } \\
\text { CHEEK }\end{array}$ & 4.81 & 1.78 & 4.97 & 1.32 & 4.49 & 1.68 & 3.46 & 1.75 \\
\hline (29) part of face & 5.76 & 1.51 & 2.60 & .90 & 5.31 & 1.30 & 5.78 & 1.60 \\
\hline $\begin{array}{l}\text { (11) impudence } \\
\text { CHINK }\end{array}$ & 4.58 & 1.59 & 3.36 & 1.18 & 5.09 & 1.16 & 3.94 & 1.79 \\
\hline (16) small opening & 4.41 & 1.64 & 5.08 & 1.28 & 4.09 & 1.82 & 4.31 & 1.66 \\
\hline (14) noise & 3.87 & 1.71 & 4.44 & 1.48 & 2.06 & .89 & 3.75 & 2.09 \\
\hline $\begin{array}{l}\text { (06) small amount of light } \\
\text { CHOP }\end{array}$ & 4.89 & 1.60 & 5.22 & 1.42 & 3.80 & 1.64 & 3.86 & 2.03 \\
\hline (23) to cut & 5.32 & 1.28 & 3.50 & 1.28 & 5.29 & 1.16 & 4.39 & 1.62 \\
\hline $\begin{array}{l}\text { (16) meat } \\
\text { CIRCLE }\end{array}$ & 5.41 & 1.46 & 3.39 & 1.04 & 4.83 & 1.61 & 5.36 & 1.57 \\
\hline (36) round shape & 6.39 & 1.09 & 2.29 & .61 & 6.17 & 1.06 & 5.56 & 1.69 \\
\hline $\begin{array}{l}\text { (04) go round/surround } \\
\text { CIRCUIT }\end{array}$ & 3.76 & 1.90 & 3.83 & 1.09 & 4.14 & 1.42 & 3.81 & 1.73 \\
\hline (18) electrical arrangement & 4.46 & 1.83 & 6.00 & .83 & 4.40 & 1.57 & 4.44 & 1.98 \\
\hline $\begin{array}{l}\text { (16) racing/athletic track } \\
\text { CLAIM }\end{array}$ & 5.36 & 1.32 & 5.00 & 1.03 & 3.88 & 1.66 & 5.61 & 1.46 \\
\hline (25) application/demand & 3.65 & 1.95 & 5.42 & .89 & 4.86 & 1.18 & 4.08 & 1.79 \\
\hline (09) ownership & 2.89 & 1.72 & 4.94 & 1.05 & 4.09 & 1.46 & 3.42 & 1.83 \\
\hline $\begin{array}{l}\text { (06) purport } \\
\text { CLASH }\end{array}$ & 2.89 & 1.74 & 4.56 & 1.17 & 4.35 & 1.49 & 3.11 & 1.76 \\
\hline (16) conflict & 4.37 & 1.93 & 4.81 & .97 & 4.77 & 1.33 & 4.25 & 1.98 \\
\hline (10) to smash together & 5.00 & 1.64 & 3.94 & 1.20 & 3.66 & 1.45 & 4.83 & 1.74 \\
\hline (08) discordant noise & 4.30 & 1.78 & 4.03 & 1.19 & 4.17 & 1.34 & 4.25 & 1.75 \\
\hline (06) pop group & 4.17 & 2.33 & 7.00 & .00 & 3.60 & 1.96 & 4.47 & 2.33 \\
\hline
\end{tabular}




\begin{tabular}{|c|c|c|c|c|c|c|c|c|}
\hline \multirow[b]{2}{*}{ Word and Meaning } & \multicolumn{2}{|c|}{ Imagery } & \multicolumn{2}{|c|}{ Age of Acquisition } & \multicolumn{2}{|c|}{ Familiarity } & \multicolumn{2}{|c|}{ Concreteness } \\
\hline & Mean & SD & Mean & SD & Mean & SD & Mean & SD \\
\hline \multicolumn{9}{|l|}{ CLASP } \\
\hline (22) to hold & 5.44 & $\begin{array}{l}1.40 \\
1.59\end{array}$ & 3.94 & 1.15 & 3.94 & 1.45 & 4.33 & 1.73 \\
\hline (12) fastener & 5.19 & 1.59 & 3.61 & 1.19 & 4.60 & 1.50 & 5.14 & 1.97 \\
\hline $\begin{array}{l}\text { (05) hands together } \\
\text { CLINK }\end{array}$ & 5.35 & 1.38 & 3.47 & 1.21 & 4.51 & 1.44 & 5.03 & 1.69 \\
\hline (28) noise & 4.83 & 1.57 & 3.64 & 1.32 & 3.97 & 1.58 & 4.36 & 1.73 \\
\hline (11) jail & 4.03 & 1.81 & 5.61 & 1.09 & 2.54 & 1.27 & 3.94 & 2.11 \\
\hline \multicolumn{9}{|l|}{ CLOSE } \\
\hline (19) near & 4.08 & 1.76 & 2.89 & .82 & 6.06 & 1.01 & 3.69 & 1.85 \\
\hline (16) to shut & 4.94 & 1.60 & 2.46 & .84 & 6.26 & 1.08 & 4.94 & 1.78 \\
\hline (05) passage/cul-de-sac & 4.84 & 1.92 & 5.14 & 1.48 & 4.37 & 1.24 & 4.97 & 1.99 \\
\hline \multicolumn{9}{|l|}{ COACH } \\
\hline (24) single deck bus & 5.95 & 1.21 & 3.72 & 1.07 & 4.94 & 1.51 & 5.72 & 1.59 \\
\hline (11) to train/teach & 4.42 & 1.38 & 4.83 & 1.01 & 4.46 & 1.25 & 4.11 & 1.71 \\
\hline (04) vehicle with horses & 5.78 & 1.51 & 3.28 & 1.02 & 3.63 & 1.79 & 4.92 & 1.86 \\
\hline \multicolumn{9}{|l|}{ COLD } \\
\hline (32) not hot & 5.19 & 2.09 & 2.03 & .77 & 6.43 & .97 & 4.53 & 1.92 \\
\hline (07) illness & 5.35 & 1.38 & 2.89 & 1.04 & 5.29 & 1.39 & 4.92 & 1.53 \\
\hline \multicolumn{9}{|l|}{ COLOGNE } \\
\hline (33) perfume & 5.25 & 1.61 & 5.53 & 1.28 & 4.46 & 1.52 & 5.67 & 1.35 \\
\hline (07) town in Germany & 3.68 & 2.08 & 5.46 & 1.16 & 3.86 & 1.40 & 4.83 & 2.17 \\
\hline \multicolumn{9}{|l|}{ COLUMN } \\
\hline (21) written vertical row & 4.68 & 1.79 & 4.22 & .95 & 5.06 & 1.31 & 4.69 & 1.81 \\
\hline (15) pillar & 6.03 & 1.05 & 4.22 & 1.03 & 4.74 & 1.68 & 5.53 & 1.71 \\
\hline \multicolumn{9}{|l|}{ COMBINATION } \\
\hline (35) that joined together & 3.67 & 1.86 & 5.00 & 1.03 & 4.71 & 1.54 & 4.39 & 1.64 \\
\hline (04) secret sequence & 3.24 & 1.48 & 5.29 & 1.26 & 3.91 & 1.34 & 3.22 & 1.83 \\
\hline COMMERCIAL & & & & & 4.51 & 1.48 & 3.53 & 1.80 \\
\hline $\begin{array}{l}\text { (22) business/buying-selling } \\
\text { (16) advertisement }\end{array}$ & $\begin{array}{l}3.30 \\
5.03\end{array}$ & $\begin{array}{l}1.72 \\
1.81\end{array}$ & $\begin{array}{l}5.89 \\
4.89\end{array}$ & $\begin{array}{r}.91 \\
1.10\end{array}$ & $\begin{array}{l}4.31 \\
4.83\end{array}$ & $\begin{array}{l}1.48 \\
1.34\end{array}$ & $\begin{array}{l}3.53 \\
4.72\end{array}$ & $\begin{array}{l}1.00 \\
1.61\end{array}$ \\
\hline $\begin{array}{l}\text { (16) advertisement } \\
\text { COMPANY }\end{array}$ & & & & & & & & \\
\hline (18) to be with friends & 4.87 & 1.77 & 4.49 & .94 & 5.54 & 1.16 & 4.78 & 1.89 \\
\hline (12) group of people/soldiers & 5.17 & 1.50 & 4.66 & 1.09 & .4 .29 & 1.58 & 4.81 & 1.58 \\
\hline (09) business concern & 4.11 & 1.65 & 4.97 & 1.17 & 4.89 & 1.43 & 4.11 & 1.76 \\
\hline CONDITION & & & & & & & & \\
\hline (29) state of & 3.51 & 1.91 & 4.58 & .95 & 5.69 & 1.17 & 4.00 & 2.08 \\
\hline (10) stipulation/rule & 3.08 & 1.91 & 4.81 & 1.17 & 5.20 & 1.33 & 3.47 & 1.83 \\
\hline CONE & & & & & & & & \\
\hline (19) container for ice cream & 5.97 & 1.14 & 2.53 & .93 & 5.17 & 1.58 & 5.97 & 1.46 \\
\hline (15) pointed shape & 5.19 & 1.67 & 4.14 & 1.23 & 4.66 & $\begin{array}{l}1.55 \\
174\end{array}$ & $\begin{array}{l}5.31 \\
5.36\end{array}$ & 1.79 \\
\hline $\begin{array}{l}\text { (05) fruit of pine tree } \\
\text { CONFLICT }\end{array}$ & 5.51 & 1.27 & 3.33 & 1.13 & 4.34 & 1.74 & 5.36 & 1.92 \\
\hline (22) opposition & 3.71 & 1.50 & 5.19 & 1.05 & 4.60 & 1.27 & 4.03 & 1.86 \\
\hline (13) battle & 4.78 & 1.77 & 4.94 & 1.04 & 4.49 & 1.20 & 4.17 & 1.64 \\
\hline CONSIDERATION & & & & & & & & \\
\hline (29) thought for others & 3.56 & 1.88 & 4.89 & 1.17 & 5.63 & 1.22 & 3.72 & 2.00 \\
\hline (07) taken into account & 2.84 & 2.02 & 5.22 & 1.11 & 5.34 & 1.53 & 3.78 & 2.06 \\
\hline CONSTITUTION & & & & & 3.91 & 1.76 & 3.28 & \\
\hline (28) set of principles & 3.11 & 1.77 & 6.51 & $\begin{array}{l}.65 \\
.81\end{array}$ & 3.31 & 1.64 & 2.97 & $\begin{array}{l}1.09 \\
1.68\end{array}$ \\
\hline $\begin{array}{l}\text { (04) physical state of health } \\
\text { CONTENT }\end{array}$ & 2.84 & 1.60 & 6.19 & & & & & \\
\hline (30) happy & 5.14 & 1.88 & 3.67 & 1.13 & 5.68 & 1.02 & 4.22 & 2.17 \\
\hline (09) that contained & 3.00 & 1.93 & 4.80 & 1.04 & 4.71 & 1.47 & 3.47 & 1.95 \\
\hline COOLNESS & & & & & & & & \\
\hline (26) lack of warmth & 4.35 & 1.92 & 3.94 & 1.26 & 5.09 & 1.40 & 4.25 & 1.83 \\
\hline (06) tranquility & 4.31 & 2.11 & 5.60 & .99 & 3.20 & 1.56 & 2.69 & 1.66 \\
\hline (04) fresh & 4.78 & 1.73 & 4.64 & 1.27 & 3.97 & 1.58 & 3.67 & 1.81 \\
\hline COPPER & & & & & & & & \\
\hline (27) metal & 5.57 & 1.73 & 4.42 & 1.19 & 5.46 & 1.46 & 5.97 & 1.32 \\
\hline (08) policeman & 5.87 & 1.26 & 4.08 & 1.21 & 3.46 & 1.70 & 5.14 & 1.92 \\
\hline (04) coin & 5.11 & 1.63 & 3.83 & 1.14 & 3.57 & 1.50 & 5.67 & 1.63 \\
\hline CORE & & & & & & & & \\
\hline (19) apple centre & 5.92 & 1.22 & 2.81 & .94 & 5.23 & 1.38 & 5.75 & 1.53 \\
\hline (17) centre & 4.16 & 1.78 & 4.86 & 1.25 & 4.69 & 1.58 & 4.46 & 1.89 \\
\hline $\begin{array}{l}\text { CORRESPONDENT } \\
\text { (25) person written to }\end{array}$ & 4.19 & 1.89 & 5.60 & .90 & 4.37 & 1.73 & 5.03 & 1.34 \\
\hline
\end{tabular}




\begin{tabular}{|c|c|c|c|c|c|c|c|c|}
\hline \multirow[b]{2}{*}{ Word and Meaning } & \multicolumn{2}{|c|}{ Imagery } & \multicolumn{2}{|c|}{ Age of Acquisition } & \multicolumn{2}{|c|}{ Familiarity } & \multicolumn{2}{|c|}{ Concreteness } \\
\hline & Mean & SD & Mean & SD & Mean & SD & Mean & SD \\
\hline $\begin{array}{l}\text { (15) journalist } \\
\text { COTTON }\end{array}$ & 4.70 & 1.47 & 5.47 & .87 & 4.26 & 1.57 & 4.92 & 1.74 \\
\hline (22) cloth & 5.89 & 1.31 & 3.66 & 1.17 & 5.89 & 1.04 & 6.19 & 1.08 \\
\hline (10) type of plant & 4.06 & 1.89 & 4.28 & 1.10 & 3.74 & 1.59 & 4.72 & 2.00 \\
\hline $\begin{array}{l}\text { (08) thread } \\
\text { COUNTRY }\end{array}$ & 5.92 & 1.48 & 3.72 & 1.30 & 4.46 & 1.79 & 5.56 & 1.69 \\
\hline (23) nation & 4.95 & 1.64 & 3.19 & 1.00 & 5.66 & 1.49 & 5.19 & 1.52 \\
\hline $\begin{array}{l}\text { (17) rural } \\
\text { COVER }\end{array}$ & 5.78 & 1.28 & 3.44 & 1.27 & 5.37 & 1.49 & 4.56 & 1.77 \\
\hline (12) material on bed & 5.33 & 1.51 & 3.03 & 1.17 & 4.80 & 1.62 & 5.19 & 1.97 \\
\hline (12) to hide & 4.46 & 1.79 & 3.19 & 1.17 & 4.86 & 1.55 & 3.92 & 1.88 \\
\hline (10) a screen/lid & 4.73 & 1.73 & 3.28 & 1.12 & 5.26 & 1.23 & 5.86 & 1.29 \\
\hline $\begin{array}{l}\text { (05) protective surround, e.g., on book } \\
\text { CRAFT }\end{array}$ & 5.44 & 1.46 & 3.56 & 1.04 & 5.40 & 1.34 & 5.83 & 1.12 \\
\hline (22) manual art & 5.22 & 1.73 & 4.66 & .89 & 4.83 & 1.52 & 4.61 & 1.69 \\
\hline $\begin{array}{l}\text { (13) ship/boat } \\
\text { CROCK }\end{array}$ & 5.44 & 1.40 & 4.56 & 1.17 & 3.83 & 1.38 & 5.19 & 2.00 \\
\hline (21) old object/person & 3.60 & 2.05 & 5.53 & 1.24 & 2.49 & 1.44 & 4.14 & 2.14 \\
\hline $\begin{array}{l}\text { (08) plates, earthenware, etc. } \\
\text { CROOK }\end{array}$ & 4.78 & 1.87 & 5.08 & 1.26 & 3.09 & 1.68 & 5.22 & 1.99 \\
\hline (30) criminal & 5.32 & 1.65 & 3.49 & 1.00 & 4.83 & 1.61 & 5.67 & 1.53 \\
\hline $\begin{array}{l}\text { (10) stick } \\
\text { CUE }\end{array}$ & 5.03 & 1.48 & 4.46 & 1.52 & 2.91 & 1.27 & 4.78 & 2.12 \\
\hline (26) rod for billiards & 5.84 & 1.55 & 5.14 & 1.10 & 4.57 & 1.34 & 5.58 & 1.55 \\
\hline (07) signal for actor & 4.73 & 1.75 & 5.61 & 1.06 & 4.63 & 1.59 & 4.53 & 1.69 \\
\hline $\begin{array}{l}\text { (07) signal to observer } \\
\text { CULT }\end{array}$ & 4.14 & 1.56 & 5.31 & .97 & 4.43 & 1.52 & 4.00 & 1.81 \\
\hline (16) group of one conviction & 3.95 & 1.80 & 6.42 & .68 & 4.43 & 1.93 & 4.61 & 1.89 \\
\hline (13) practice/style & 2.89 & 1.39 & 6.47 & .60 & 3.80 & 1.72 & 3.47 & 1.79 \\
\hline $\begin{array}{l}\text { (07) system of religious worship } \\
\text { CURSE }\end{array}$ & 4.65 & 1.83 & 5.94 & .91 & 4.97 & 1.50 & 4.39 & 1.85 \\
\hline (25) oath/swear & 4.60 & 1.81 & 4.37 & 1.04 & 5.09 & 1.50 & 4.33 & 1.56 \\
\hline $\begin{array}{l}\text { (14) wish bad fortune } \\
\text { DART }\end{array}$ & 4.38 & 1.46 & 4.14 & 1.34 & 4.83 & 1.52 & 4.14 & 1.93 \\
\hline (32) missile/pastime & 5.92 & 1.36 & 3.92 & 1.16 & 4.89 & 1.77 & 5.78 & 1.46 \\
\hline $\begin{array}{l}\text { (08) fast motion } \\
\text { DATE }\end{array}$ & 4.43 & 1.69 & 4.77 & .90 & 3.89 & 1.28 & 3.89 & 1.54 \\
\hline (29) time of year & 4.78 & 1.65 & 2.83 & .76 & 6.21 & .99 & 4.72 & 2.22 \\
\hline (06) fruit & 5.49 & 1.57 & 3.92 & 1.06 & 4.11 & 1.69 & 4.97 & 2.14 \\
\hline $\begin{array}{l}\text { (05) appointment } \\
\text { DEAL }\end{array}$ & 4.22 & 1.76 & 4.78 & 1.20 & 4.63 & 1.66 & 3.58 & 1.85 \\
\hline $\begin{array}{l}\text { (19) agreement } \\
\text { (16) hand of cards }\end{array}$ & $\begin{array}{l}3.41 \\
5.54\end{array}$ & $\begin{array}{l}1.62 \\
1.15\end{array}$ & $\begin{array}{l}4.58 \\
4.11\end{array}$ & $\begin{array}{r}.98 \\
1.22\end{array}$ & $\begin{array}{l}4.63 \\
4.54\end{array}$ & $\begin{array}{l}1.44 \\
1.48\end{array}$ & $\begin{array}{l}3.50 \\
4.72\end{array}$ & $\begin{array}{l}1.82 \\
1.95\end{array}$ \\
\hline $\begin{array}{l}\text { (04) to share out } \\
\text { DECK }\end{array}$ & 5.05 & 1.64 & 4.03 & 1.19 & 4.60 & 1.31 & 3.97 & 1.59 \\
\hline (35) platform on ship & 5.89 & 1.07 & 3.33 & 1.03 & 4.89 & 1.67 & 6.19 & 1.10 \\
\hline $\begin{array}{l}\text { (05) collection of cards } \\
\text { DEGREE }\end{array}$ & 5.28 & 1.74 & 4.51 & 1.16 & 3.49 & 1.61 & 4.64 & 2.03 \\
\hline (15) temperature & 3.54 & 2.06 & 4.51 & .97 & 5.26 & 1.18 & 4.31 & 1.78 \\
\hline (14) stage in university & 4.89 & 1.89 & 5.72 & 1.22 & 5.57 & 1.29 & 4.47 & 1.85 \\
\hline (06) measure of angle & 4.43 & 2.09 & 5.03 & .96 & 4.51 & 1.36 & 4.28 & 1.77 \\
\hline $\begin{array}{l}\text { (05) measure/level } \\
\text { DELTA }\end{array}$ & 3.24 & 1.84 & 5.56 & 1.04 & 5.03 & 1.42 & 2.97 & 1.72 \\
\hline (21) river mouth & 4.32 & 2.13 & 5.69 & .95 & 3.89 & 1.92 & 5.47 & 1.44 \\
\hline $\begin{array}{l}\text { (15) Greek letter } \\
\text { DEPARTMENT }\end{array}$ & 4.72 & 1.97 & 6.17 & .80 & 4.26 & 1.84 & 4.63 & 2.14 \\
\hline (26) section of an institution & 4.38 & 1.58 & 5.47 & 1.09 & 5.77 & 1.29 & 4.83 & 1.59 \\
\hline $\begin{array}{l}\text { (11) type of shop/store } \\
\text { DESIGN }\end{array}$ & 5.23 & 1.55 & 4.26 & .77 & 4.86 & 1.33 & 5.17 & 1.83 \\
\hline (30) pattern & 5.30 & 1.61 & 3.94 & 1.05 & 5.91 & 1.03 & 4.97 & 1.89 \\
\hline (06) to create & 4.14 & 1.96 & 4.83 & .99 & 4.94 & 1.39 & 3.92 & 1.62 \\
\hline $\begin{array}{l}\text { (04) to cause to happen } \\
\text { DEVELOPMENT }\end{array}$ & 2.57 & 1.36 & 5.20 & 1.12 & 3.63 & 1.49 & 2.61 & 1.40 \\
\hline (28) progressive change & 3.25 & 1.80 & 5.58 & .89 & 5.37 & 1.33 & 3.64 & 1.83 \\
\hline $\begin{array}{l}\text { (11) area of new building } \\
\text { DEVICE }\end{array}$ & 4.58 & 1.89 & 5.47 & .80 & 4.17 & 1.34 & 4.53 & 1.95 \\
\hline (34) tool & 4.32 & 1.79 & 4.89 & 1.07 & 4.63 & 1.29 & 5.08 & 1.91 \\
\hline
\end{tabular}




\begin{tabular}{|c|c|c|c|c|c|c|c|c|}
\hline \multirow[b]{2}{*}{ Word and Meaning } & \multicolumn{2}{|c|}{ Imagery } & \multicolumn{2}{|c|}{ Age of Acquisition } & \multicolumn{2}{|c|}{ Familiarity } & \multicolumn{2}{|c|}{ Concreteness } \\
\hline & Mean & $\mathrm{SD}$ & Mean & SD & Mean & SD & Mean & SD \\
\hline (05) method & 2.92 & 1.91 & 5.31 & 1.17 & 3.79 & 1.55 & 3.43 & 1.71 \\
\hline \multicolumn{9}{|l|}{ DIET } \\
\hline $\begin{array}{l}\text { (27) to restrict eating } \\
\text { (13) kind of food eaten }\end{array}$ & 5.03 & $\begin{array}{l}1.67 \\
1.44\end{array}$ & $\begin{array}{l}4.74 \\
5.25\end{array}$ & $\begin{array}{l}1.25 \\
1.21\end{array}$ & $\begin{array}{l}5.77 \\
5.43\end{array}$ & $\begin{array}{l}1.24 \\
1.52\end{array}$ & $\begin{array}{l}4.89 \\
4.83\end{array}$ & $\begin{array}{l}1.84 \\
1.57\end{array}$ \\
\hline \multicolumn{9}{|l|}{$\begin{array}{l}\text { (13) kind of food eaten } \\
\text { DIFFERENCE }\end{array}$} \\
\hline (31) unlikeness & 3.19 & 2.05 & 3.64 & 1.06 & 5.89 & 1.17 & 4.17 & 2.26 \\
\hline (04) subtraction & 2.86 & 1.62 & 4.09 & 1.16 & 3.97 & 1.56 & 3.11 & 1.85 \\
\hline \multicolumn{9}{|l|}{ DINER } \\
\hline $\begin{array}{l}\text { (35) one who eats } \\
\text { (04) restaurant in train }\end{array}$ & $\begin{array}{l}5.44 \\
4.76\end{array}$ & $\begin{array}{l}1.64 \\
1.90\end{array}$ & 4.64 & 1.06 & 4.26 & 1.86 & 5.75 & 1.19 \\
\hline \multicolumn{2}{|l|}{ DIP } & 1.90 & 5.42 & 1.09 & 2.97 & 1.58 & 4.56 & 1.95 \\
\hline (23) immerse briefly & 5.27 & 1.41 & 3.42 & 1.09 & 5.37 & 1.22 & 5.03 & 1.46 \\
\hline (14) bathe & 5.00 & 1.45 & 4.00 & 1.03 & 3.54 & 1.30 & 4.28 & 1.52 \\
\hline \multicolumn{9}{|l|}{$\begin{array}{l}\text { (14) bathe } \\
\text { DIRECTION }\end{array}$} \\
\hline (35) course of movement & 3.78 & 1.88 & 3.81 & .94 & 5.80 & 1.21 & 3.78 & 1.84 \\
\hline$(05)$ instruction & 3.30 & 1.39 & 4.58 & .98 & 4.86 & 1.33 & 3.58 & 1.67 \\
\hline \multicolumn{9}{|l|}{ DISC } \\
\hline $\begin{array}{l}\text { (25) record } \\
\text { (11) round flat plate }\end{array}$ & 5.80 & 1.55 & $\begin{array}{l}4.74 \\
458\end{array}$ & $\begin{array}{l}1.16 \\
1.04\end{array}$ & $\begin{array}{l}5.03 \\
4.29\end{array}$ & $\begin{array}{l}1.59 \\
1.41\end{array}$ & $\begin{array}{l}0.06 \\
4.94\end{array}$ & $\begin{array}{l}1.67 \\
1.73\end{array}$ \\
\hline $\begin{array}{l}\text { (11) round lat prate } \\
\text { (04) bone }\end{array}$ & $\begin{array}{l}4.32 \\
3.40\end{array}$ & 1.60 & $\begin{array}{l}4.58 \\
5.72\end{array}$ & $\begin{array}{l}1.04 \\
1.02\end{array}$ & 2.69 & 1.75 & 4.31 & \\
\hline \multicolumn{9}{|l|}{$\begin{array}{l}\text { (04) bone } \\
\text { DISCHARGE }\end{array}$} \\
\hline (16) secrete/emit & 4.11 & 1.96 & 5.89 & .88 & 4.57 & 1.50 & 4.33 & 1.73 \\
\hline (16) release & 3.70 & 1.89 & 5.43 & 1.08 & 4.37 & 1.53 & 4.31 & 1.52 \\
\hline (06) dismiss & 3.53 & 1.66 & 5.17 & 1.07 & 4.14 & 1.36 & 3.31 & 1.66 \\
\hline \multicolumn{9}{|l|}{ DISCORD } \\
\hline (20) musical disharmony & 3.97 & 1.98 & 5.69 & 1.17 & 4.03 & 1.70 & 3.56 & 1.77 \\
\hline (14) strife & 3.24 & 1.91 & 6.19 & .74 & 3.77 & 1.79 & 3.06 & 1.87 \\
\hline DIVISION & & & & & & & & \\
\hline (20) part/separation & 3.54 & 1.57 & 4.47 & 1.14 & 4.51 & 1.38 & 3.67 & 1.63 \\
\hline (10) arithmetic operation & 4.19 & 1.89 & 3.47 & 1.01 & 5.34 & 1.51 & 3.94 & 1.90 \\
\hline (07) section of league & 2.87 & 1.91 & 4.97 & .99 & 3.91 & 1.68 & 3.17 & 1.68 \\
\hline DRAIN & & & & & & & & \\
\hline (31) for waste liquid & 5.54 & 1.46 & 3.54 & 1.20 & 5.31 & 1.26 & 5.78 & 1.75 \\
\hline (08) take liquid from solid & 4.49 & 1.67 & 4.39 & 1.30 & 4.97 & 1.54 & 4.19 & 1.82 \\
\hline DRAPE & & 150 & 5.00 & 1.13 & 4.00 & 1.79 & 4.56 & \\
\hline $\begin{array}{l}\text { (24) arrange material } \\
\text { (15) curtain }\end{array}$ & $\begin{array}{l}4.41 \\
5.32\end{array}$ & $\begin{array}{l}1.50 \\
1.73\end{array}$ & $\begin{array}{l}5.00 \\
4.94\end{array}$ & 1.47 & 3.11 & 1.74 & 5.33 & $\begin{array}{l}1.69 \\
1.56\end{array}$ \\
\hline $\begin{array}{l}\text { (15) curtain } \\
\text { DRESS }\end{array}$ & 5.32 & & & & & & & 1.56 \\
\hline $\begin{array}{l}\text { DRESS } \\
\text { (34) female clothing }\end{array}$ & 6.11 & 1.49 & 2.61 & .98 & 5.77 & 1.35 & 6.08 & 1.30 \\
\hline (06) to put on clothes & 5.49 & 1.50 & 2.14 & .86 & 6.23 & 1.27 & 5.14 & 1.55 \\
\hline DRINK & & & & & & & & \\
\hline (32) liquid refreshment & 5.61 & 1.40 & 2.14 & 1.08 & 6.51 & .81 & 6.00 & 1.08 \\
\hline (08) to swallow liquid & 5.89 & 1.24 & 2.03 & .87 & 6.40 & .80 & 5.53 & 1.48 \\
\hline DROVE & & & & & & & & \\
\hline (21) herd & 4.27 & 1.73 & 5.08 & 1.09 & 2.94 & 1.39 & 4.17 & 1.89 \\
\hline (16) past of "drive" & 3.35 & 2.15 & 3.69 & 1.26 & 5.69 & 1.45 & 3.64 & 2.07 \\
\hline DUMP & & & & .84 & & & & 1.76 \\
\hline (29) refuse area & $\begin{array}{l}5.69 \\
467\end{array}$ & 1.33 & $\begin{array}{l}3.77 \\
3.67\end{array}$ & $\begin{array}{l}.84 \\
.91\end{array}$ & $\begin{array}{l}3.40 \\
5.20\end{array}$ & $\begin{array}{l}1.22 \\
1.24\end{array}$ & $\begin{array}{l}3.31 \\
4.33\end{array}$ & 1.62 \\
\hline $\begin{array}{l}\text { (09) to deposit } \\
\text { EARTH }\end{array}$ & & & & & & & & \\
\hline (23) world & 5.46 & 1.67 & 3.25 & 1.04 & 5.37 & 1.53 & 5.22 & 2.00 \\
\hline (17) soil & 5.80 & 1.47 & 3.06 & 1.15 & 5.34 & 1.37 & 6.22 & .98 \\
\hline EASE & & & & & & & & \\
\hline (24) without difficulty & 3.73 & 1.78 & 4.06 & .98 & 5.51 & 1.30 & 3.83 & 2.12 \\
\hline (11) leisure & 3.81 & 1.81 & 4.83 & 1.03 & 4.09 & 1.48 & 3.44 & 1.38 \\
\hline (05) remove carefully & 3.57 & 1.81 & 4.79 & .99 & 3.46 & 1.25 & 3.42 & 1.38 \\
\hline EFFECT & & & & & & & & \\
\hline (28) result & 3.03 & 1.80 & 4.67 & 1.03 & 5.06 & 1.45 & 4.06 & 1.72 \\
\hline $\begin{array}{l}\text { (04) impact on } \\
\text { ENSEMBLE }\end{array}$ & 3.38 & 1.95 & 4.60 & 1.10 & 5.40 & 1.15 & 3.89 & 1.70 \\
\hline $\begin{array}{l}\text { ENSEMBLE } \\
\text { (17) musical group }\end{array}$ & 5.11 & 1.76 & 6.03 & .96 & 3.83 & 1.86 & 4.92 & 1.88 \\
\hline $\begin{array}{l}\text { (09) meeting } \\
\text { (1) }\end{array}$ & 4.17 & 1.80 & 5.89 & .84 & 3.29 & 1.63 & 4.11 & 1.84 \\
\hline (08) small group & 4.33 & 1.76 & 5.97 & .87 & 3.31 & 1.35 & 4.42 & 1.74 \\
\hline ESTABLISHMENT & & & & & & & & \\
\hline (23) governing body/institution & 3.89 & 1.67 & 6.06 & .86 & 4.06 & 1.53 & 4.17 & 1.91 \\
\hline (09) a building & 4.39 & 1.99 & 5.58 & .83 & 4.06 & 1.55 & 5.11 & 1.58 \\
\hline (07) conventional society & 3.58 & 1.88 & 6.06 & .97 & 4.11 & 1.70 & 3.61 & 1.88 \\
\hline
\end{tabular}




\begin{tabular}{|c|c|c|c|c|c|c|c|c|}
\hline \multirow[b]{2}{*}{ Word and Meaning } & \multicolumn{2}{|c|}{ Imagery } & \multicolumn{2}{|c|}{ Age of Acquisition } & \multicolumn{2}{|c|}{ lamiliarity } & \multicolumn{2}{|c|}{ Concreteness } \\
\hline & Mean & SD & Mean & SD & Mean & $\mathrm{SD}$ & Mean & SD \\
\hline \multicolumn{9}{|l|}{ EXCHANGE } \\
\hline (34) swap & 3.73 & 1.93 & 3.86 & 1.16 & 5.80 & 1.21 & 4.28 & 1.79 \\
\hline (05) stocks and shares & 3.24 & 1.76 & 6.31 & .79 & 3.46 & 1.52 & 3.64 & 1.70 \\
\hline \multicolumn{9}{|l|}{ EXCUSE } \\
\hline (28) reason & 3.25 & 1.91 & 3.86 & 1.06 & 5.54 & 1.34 & 3.44 & 1.72 \\
\hline (06) pardon & 3.17 & 1.66 & 3.83 & 1.28 & 4.80 & 1.64 & 3.74 & 1.78 \\
\hline (04) to exempt & 3.14 & 1.86 & 4.53 & 1.14 & 4.60 & 1.61 & 3.25 & 1.61 \\
\hline \multicolumn{9}{|l|}{ EXHAUST } \\
\hline (14) fatigue & 4.27 & $\begin{array}{l}1.00 \\
1.62\end{array}$ & $\begin{array}{l}4.39 \\
4.57\end{array}$ & $\begin{array}{l}1.14 \\
1.25\end{array}$ & $\begin{array}{l}5.49 \\
5.03\end{array}$ & $\begin{array}{l}1.34 \\
1.40\end{array}$ & $\begin{array}{l}4.92 \\
4.22\end{array}$ & $\begin{array}{l}1.75 \\
1.93\end{array}$ \\
\hline \multicolumn{7}{|l|}{ EXPERIENCE } & & 1.52 \\
\hline (33) effect by event/knowledge & 3.06 & 1.97 & 5.00 & 1.07 & 5.43 & 1.44 & 3.97 & 2.15 \\
\hline $\begin{array}{l}\text { (06) happening } \\
\text { EXPRESS }\end{array}$ & EXPRESS & 1.83 & 4.75 & .83 & 5.20 & 1.43 & 3.67 & 2.07 \\
\hline (26) fast vehicle & 4.72 & 1.43 & 4.83 & 1.26 & 4.23 & 1.59 & 5.08 & 1.69 \\
\hline (09) newspaper & 5.11 & 1.74 & 5.25 & 1.21 & 4.26 & 1.65 & 4.42 & 2.18 \\
\hline $\begin{array}{l}\text { (05) method of statement } \\
\text { FACTOR }\end{array}$ & & 1.81 & 5.39 & 1.06 & 4.29 & 1.65 & 3.60 & 1.71 \\
\hline (33) element & 2.78 & 1.93 & 5.71 & 1.00 & 4.60 & 1.38 & 3.75 & 1.91 \\
\hline $\begin{array}{l}\text { (04) numerical divisor } \\
\text { FAIRY }\end{array}$ & \multicolumn{7}{|c|}{ FAIRY } & \\
\hline (32) my thical creature & 5.67 & 1.47 & 2.42 & 1.01 & 4.97 & 1.93 & 3.92 & 2.43 \\
\hline (04) brand of soap & 5.19 & 1.81 & 4.89 & 1.17 & 3.26 & 1.54 & 4.53 & 2.25 \\
\hline \multicolumn{9}{|l|}{ FAT } \\
\hline (20) overweight & 6.08 & 1.06 & 2.72 & 1.02 & 6.00 & 1.15 & 5.33 & 1.68 \\
\hline \multicolumn{9}{|l|}{ FAWN } \\
\hline (34) young deer & 5.87 & 1.34 & 3.97 & 1.16 & 4.23 & 1.64 & 5.58 & 1.69 \\
\hline \multicolumn{9}{|l|}{ FEELING } \\
\hline (31) sense or emotion & 4.62 & 1.98 & 3.67 & 1.25 & 6.26 & .81 & 4.64 & 2.26 \\
\hline \multicolumn{9}{|l|}{ FELLOW } \\
\hline (21) man & 5.00 & 2.04 & 4.74 & 1.34 & 4.60 & 1.61 & 5.50 & 1.34 \\
\hline \multicolumn{9}{|l|}{ FENCING } \\
\hline (24) sport & 5.54 & 1.54 & 4.92 & 1.04 & 4.14 & 1.66 & 5.06 & 1.56 \\
\hline \multicolumn{9}{|l|}{ FIDDLE } \\
\hline (35) violin & 6.33 & 1.13 & 3.89 & 1.15 & 4.57 & 1.64 & 5.64 & 1.81 \\
\hline \multicolumn{9}{|l|}{$\begin{array}{l}\text { (04) dishonest practice } \\
\text { FILLING }\end{array}$} \\
\hline (21) that inserted & 4.05 & 1.87 & 3.94 & 1.10 & 4.85 & 1.31 & 4.44 & 1.57 \\
\hline $\begin{array}{l}\text { (16) dental filling } \\
\text { FLIES }\end{array}$ & \multicolumn{8}{|c|}{ FLIES } \\
\hline (34) insects & 6.41 & .94 & 2.50 & .73 & 5.77 & 1.31 & 6.25 & 1.21 \\
\hline $\begin{array}{l}\text { (04) fastener on trousers } \\
\text { FLORA }\end{array}$ & 5.57 & 1.52 & 5.08 & 1.38 & 4.34 & 1.43 & 4.83 & 2.30 \\
\hline (33) foliage & 4.43 & 1.95 & 6.26 & .91 & 3.54 & 1.68 & 4.61 & 2.18 \\
\hline $\begin{array}{l}\text { (07) margarine } \\
\text { FLOUR ISH }\end{array}$ & 5.60 & 1.62 & 6.51 & 1.00 & 4.49 & 1.80 & 5.14 & 2.24 \\
\hline (28) thrive & 4.08 & 1.81 & 5.25 & 1.06 & 4.74 & 1.70 & 3.89 & 1.71 \\
\hline $\begin{array}{l}\text { (11) ornamental expression } \\
\text { FLUTTER }\end{array}$ & 3.19 & 2.14 & 6.06 & .91 & 2.71 & 1.47 & 2.86 & 1.38 \\
\hline (16) flying/flap wings & 5.31 & 1.56 & 3.86 & 1.36 & 4.23 & 1.71 & 5.11 & 1.26 \\
\hline (10) uneven movement & 4.05 & 1.77 & 4.40 & 1.13 & 3.86 & 1.42 & 3.97 & 1.66 \\
\hline $\begin{array}{l}(08) \text { bet } \\
\text { FOLLOWING }\end{array}$ & 2.89 & 1.59 & 6.23 & .90 & 2.47 & 1.29 & 2.83 & 1.77 \\
\hline (27) coming later & 2.92 & 1.75 & 3.64 & 1.03 & 5.71 & 1.16 & 3.81 & 2.08 \\
\hline $\begin{array}{l}\text { (12) supporters } \\
\text { FOOTBALL }\end{array}$ & 4.50 & 1.59 & 5.14 & .96 & 4.09 & 1.52 & 4.39 & 1.42 \\
\hline (36) game & 5.87 & 1.40 & 2.78 & .75 & 6.17 & 1.03 & 5.75 & 1.42 \\
\hline $\begin{array}{l}\text { (04) type of ball } \\
\text { IORM }\end{array}$ & 5.78 & 1.53 & 2.60 & 1.05 & 5.29 & 1.47 & 5.72 & 1.54 \\
\hline (16) document & 5.00 & 1.61 & 4.86 & .98 & 5.23 & 1.29 & 4.83 & 1.71 \\
\hline
\end{tabular}




\begin{tabular}{|c|c|c|c|c|c|c|c|c|}
\hline \multirow[b]{2}{*}{ Word and Meaning } & \multicolumn{2}{|c|}{ Imagery } & \multicolumn{2}{|c|}{ Age of Acquisition } & \multicolumn{2}{|c|}{ Familiarity } & \multicolumn{2}{|c|}{ Concreteness } \\
\hline & Mean & SD & Mean & SD & Mean & SD & Mean & SD \\
\hline (11) shape & 3.95 & 1.87 & 4.44 & 1.07 & 4.74 & 1.52 & 4.08 & 1.72 \\
\hline (05) nature/style & 3.32 & 1.79 & 5.03 & 1.24 & 4.11 & 1.49 & 3.47 & 1.82 \\
\hline (04) bench/seat & 4.64 & 2.21 & 5.28 & 1.47 & 2.29 & 1.21 & 4.08 & 2.42 \\
\hline \multicolumn{9}{|l|}{ FORTUNE } \\
\hline (28) wealth & 5.14 & 1.58 & 3.75 & 1.16 & 5.06 & 1.37 & 4.03 & 2.01 \\
\hline (12) luck & 3.64 & 1.97 & 4.06 & 1.08 & 4.97 & 1.50 & 3.42 & 1.96 \\
\hline \multicolumn{9}{|l|}{ FOUNDATION } \\
\hline (27) base & 3.81 & 1.98 & 4.56 & 1.19 & 4.63 & 1.61 & 4.53 & 1.77 \\
\hline (05) initial part & 2.81 & 1.65 & 5.14 & 1.13 & 3.86 & 1.50 & 3.75 & 1.69 \\
\hline (05) cosmetics/make-up & 4.62 & 1.95 & 6.25 & .80 & 3.63 & 1.90 & 4.56 & 1.99 \\
\hline \multicolumn{9}{|l|}{ FUNCTION } \\
\hline (17) purpose & 2.94 & 2.07 & 5.17 & 1.04 & 5.06 & $\begin{array}{l}1.35 \\
1.39\end{array}$ & $\begin{array}{l}3.03 \\
4.83\end{array}$ & $\begin{array}{l}1.95 \\
1.50\end{array}$ \\
\hline (15) ceremony/party & 5.16 & 1.26 & 5.75 & 1.16 & 4.80 & 1.39 & 4.83 & 1.50 \\
\hline $\begin{array}{l}\text { (06) operate correctly } \\
\text { GALLERY }\end{array}$ & 3.22 & 1.55 & 5.17 & 1.09 & 4.60 & 1.50 & 3.83 & 1.77 \\
\hline (32) place for exhibition & 6.00 & 1.19 & 4.44 & 1.12 & 5.57 & 1.25 & 6.00 & 1.33 \\
\hline & \multicolumn{7}{|c|}{ GAME } & 2.13 \\
\hline (33) pastime & 5.60 & 1.42 & 2.56 & 1.12 & 5.77 & 1.20 & 5.47 & 1.48 \\
\hline (06) hunted animals & 5.25 & 1.48 & 4.44 & 1.01 & 3.89 & 1.47 & 4.47 & 1.66 \\
\hline \multicolumn{9}{|l|}{ GAS } \\
\hline (23) fuel & 4.83 & 1.81 & 3.89 & 1.26 & 5.66 & 1.39 & 5.42 & 1.46 \\
\hline \multicolumn{9}{|l|}{ GENERATION } \\
\hline (31) persons from same age range & 4.56 & 1.82 & 5.08 & 1.21 & 4.86 & 1.61 & & 1.55 \\
\hline \multicolumn{8}{|l|}{ GIG } & 1.61 \\
\hline (31) dance/concert & 5.95 & 1.29 & 6.39 & .86 & 4.83 & 1.89 & 5.64 & 1.34 \\
\hline \multicolumn{8}{|l|}{ GLARE } & 2.37 \\
\hline (22) bright light & 5.64 & 1.29 & 4.29 & 1.19 & 4.77 & 1.55 & 5.03 & 1.76 \\
\hline $\begin{array}{l}\text { (18) look at angrily } \\
\text { GOAL }\end{array}$ & 5.14 & 1.66 & 4.25 & 1.04 & 4.86 & 1.57 & 4.72 & 1.71 \\
\hline (22) aim & 3.36 & 1.75 & 5.17 & .99 & 4.69 & 1.56 & 3.67 & 2.00 \\
\hline (18) score & 5.46 & 1.76 & 3.08 & .80 & 5.31 & 1.51 & 4.83 & 1.80 \\
\hline GRAVE & & & & & & & & \\
\hline (32) ground for burying & 6.25 & .89 & 3.31 & .94 & 5.29 & 1.47 & 5.81 & 1.60 \\
\hline (08) serious & 3.68 & 1.85 & 4.40 & .93 & 4.51 & 1.46 & 3.03 & 1.91 \\
\hline GROUND & & & & & & & & \\
\hline (33) earth & 5.42 & 1.53 & 2.61 & .95 & 5.43 & 1.54 & 5.89 & 1.15 \\
\hline (06) lowest floor & 4.84 & 1.98 & 3.31 & 1.05 & 4.88 & 1.66 & 4.58 & 1.83 \\
\hline GROWTH & & & & & & & & \\
\hline (18) increase in size & 4.97 & 1.90 & 3.28 & 1.07 & 6.03 & 1.21 & 4.72 & 1.87 \\
\hline (17) development & 4.08 & 2.10 & 4.17 & 1.26 & 5.60 & 1.20 & 4.08 & 1.77 \\
\hline (04) abnormal appendage & 4.28 & 1.76 & 5.94 & 1.05 & 3.44 & 1.38 & 4.53 & 1.59 \\
\hline GUARD & & & & & & & & \\
\hline (20) to protect & 4.49 & 1.85 & 3.58 & 1.01 & 5.09 & 1.32 & 3.97 & 1.77 \\
\hline (15) soldier & 5.70 & 1.29 & 2.97 & .93 & 4.26 & 1.56 & 5.50 & 1.82 \\
\hline (04) watchman & 5.35 & 1.40 & 3.72 & 1.04 & 4.94 & 1.49 & 5.42 & 1.67 \\
\hline GUIDE & & & & & & & & \\
\hline (30) one showing the way & 5.08 & 1.65 & 4.33 & 1.11 & 4.97 & 1.30 & 5.66 & 1.41 \\
\hline (06) girl's organisation & 5.35 & 1.80 & 3.75 & .76 & 4.66 & 1.69 & 4.94 & 2.00 \\
\hline $\begin{array}{l}\text { (04) book of information } \\
\text { GUSH }\end{array}$ & 5.22 & 1.42 & 4.72 & .90 & 4.51 & 1.25 & 5.14 & 1.64 \\
\hline (29) rushing liquid & 4.97 & 1.70 & 4.17 & 1.13 & 4.37 & 1.51 & 4.61 & 1.66 \\
\hline (09) overflowing reaction & 4.47 & 1.86 & 5.06 & 1.33 & 4.17 & 1.81 & 4.31 & 1.66 \\
\hline GUY & & & & & & & & \\
\hline (33) male person & 5.94 & 1.47 & 4.75 & 1.16 & 5.69 & 1.47 & 5.81 & 1.31 \\
\hline (04) stuffed dummy & 4.81 & 1.83 & 3.57 & 1.08 & 3.31 & 1.47 & 4.83 & 1.92 \\
\hline HABIT & & & & & & & & \\
\hline (35) persistant action & 4.61 & 1.92 & 4.25 & 1.12 & 6.14 & .93 & 4.67 & 1.81 \\
\hline $\begin{array}{l}\text { (05) monk's clothing } \\
\text { HAIL }\end{array}$ & 5.47 & 1.50 & 5.03 & 1.17 & 3.26 & 1.46 & 5.25 & 2.10 \\
\hline (22) frozen vapour & 5.95 & 1.49 & 3.63 & 1.51 & 4.83 & 1.59 & 5.61 & 1.67 \\
\hline (18) greeting & 4.00 & 1.76 & 4.83 & 1.24 & 2.97 & 1.44 & 3.83 & 1.69 \\
\hline
\end{tabular}




\begin{tabular}{|c|c|c|c|c|c|c|c|c|}
\hline \multirow[b]{2}{*}{ Word and Meaning } & \multicolumn{2}{|c|}{ Imagery } & \multicolumn{2}{|c|}{ Age of Acquisition } & \multicolumn{2}{|c|}{ Familiarity } & \multicolumn{2}{|c|}{ Concreteness } \\
\hline & Mean & SD & Mean & SD & Mean & SD & Mean & SD \\
\hline \multicolumn{9}{|l|}{ HALL } \\
\hline (34) part of house & 6.00 & 1.47 & 2.58 & .92 & 5.74 & 1.27 & 5.81 & 1.51 \\
\hline \multicolumn{9}{|l|}{ HALTER } \\
\hline (27) rope/strap for animals & 5.27 & 1.77 & 5.33 & 1.25 & 3.63 & 1.79 & 5.47 & 1.91 \\
\hline $\begin{array}{l}\text { (10) type of dress } \\
\text { HEAD }\end{array}$ & \multicolumn{3}{|c|}{ HEAD } & .82 & 3.34 & 1.67 & 4.64 & 2.00 \\
\hline (33) upper part of body & 6.41 & 1.05 & 1.80 & .79 & 6.54 & .65 & 6.64 & .71 \\
\hline $\begin{array}{l}\text { (04) top of } \\
\text { HEART }\end{array}$ & 4.70 & 1.74 & 3.66 & .95 & 5.06 & 1.29 & 4.36 & 1.65 \\
\hline (33) organ of body & 5.97 & 1.28 & 3.29 & .85 & 6.11 & 1.12 & 6.42 & .95 \\
\hline \multicolumn{8}{|l|}{ HIDE } & 1.75 \\
\hline (31) conceal & 4.65 & 1.80 & 2.47 & .96 & 6.14 & .99 & 4.86 & 1.53 \\
\hline (08) skin & 4.78 & 1.77 & 4.28 & 1.04 & 3.71 & 1.45 & 4.75 & 1.94 \\
\hline \multicolumn{9}{|l|}{ HIND } \\
\hline (22) rear & 4.68 & 1.92 & 4.40 & 1.07 & 4.71 & 1.32 & 4.25 & 2.02 \\
\hline (17) female deer & 4.53 & 1.82 & 5.20 & 1.49 & 2.91 & 1.57 & 5.08 & 2.06 \\
\hline \multicolumn{9}{|l|}{ HOLD } \\
\hline (31) keep in hand & 4.89 & 1.68 & 2.64 & 1.16 & 5.77 & 1.10 & 4.64 & 1.87 \\
\hline \multicolumn{9}{|l|}{ HOP } \\
\hline (35) jump & 5.43 & 1.35 & 2.39 & .68 & 4.83 & 1.42 & 4.94 & 1.65 \\
\hline (05) beer plant & 3.03 & 1.79 & 5.53 & 1.19 & 3.71 & 1.72 & 4.58 & 2.31 \\
\hline \multicolumn{9}{|l|}{ HORN } \\
\hline (21) hard substance on head of animal & 5.89 & 1.18 & 3.20 & 1.37 & 5.20 & 1.37 & 5.58 & 1.77 \\
\hline (10) car warning system & 5.73 & 1.50 & 2.78 & .82 & 4.97 & 1.38 & 5.50 & 1.62 \\
\hline \multicolumn{8}{|l|}{ HOWLER } & 1.99 \\
\hline (18) joke & 3.47 & 1.77 & 5.83 & 1.04 & 2.97 & 1.70 & 3.33 & 1.76 \\
\hline (12) error & 2.75 & 1.75 & 6.11 & 1.05 & 2.26 & 1.23 & 2.94 & 1.41 \\
\hline \multicolumn{8}{|l|}{ HUMP } & 1.98 \\
\hline (19) bump & 4.76 & 1.75 & 3.57 & 1.10 & 4.17 & 1.65 & 4.89 & 1.73 \\
\hline (15) on camel & 5.78 & 1.42 & 2.94 & .86 & 4.60 & 1.59 & 5.44 & 1.82 \\
\hline $\begin{array}{l}\text { (05) small hill } \\
\text { IMPEDIMENT }\end{array}$ & 5.08 & 1.53 & 3.75 & 1.48 & 3.37 & 1.68 & 5.33 & 1.51 \\
\hline (20) hinderance & 4.19 & 1.97 & 5.71 & 1.06 & 4.20 & 1.72 & 3.36 & 1.86 \\
\hline (13) defect & 4.06 & 1.87 & 5.81 & 1.05 & 4.03 & 1.38 & 4.08 & 1.74 \\
\hline IMPRESSION & & & & & & & & \\
\hline (24) opinion of & 3.54 & 2.00 & 5.20 & 1.04 & 5.20 & 1.17 & 3.56 & 2.09 \\
\hline (10) dent/mark & 4.60 & 1.65 & 5.14 & 1.08 & 4.14 & 1.31 & 4.42 & 1.67 \\
\hline $\begin{array}{l}\text { (04) sound/visual effect } \\
\text { INCLINE }\end{array}$ & 3.14 & 1.93 & 5.50 & 1.01 & 4.60 & 1.40 & 3.61 & 1.78 \\
\hline (24) slope & 4.41 & 1.48 & 5.11 & 1.17 & 4.51 & 1.40 & 4.94 & 1.67 \\
\hline (08) tend towards & 3.65 & 1.71 & 5.39 & 1.04 & 4.46 & 1.36 & 3.50 & 1.54 \\
\hline (07) tilt & 4.08 & 1.48 & 5.11 & 1.17 & 4.14 & 1.46 & 3.44 & 1.46 \\
\hline INDUSTRY & & & & & 4.14 & 1.40 & 3.44 & 1.40 \\
\hline (32) manufacture & 3.95 & 2.00 & 5.42 & 1.09 & 4.74 & 1.70 & 4.11 & 1.79 \\
\hline $\begin{array}{l}\text { (06) hard work } \\
\text { INTEREST }\end{array}$ & 3.78 & 1.72 & 5.50 & .96 & 3.60 & 1.34 & 3.56 & 1.50 \\
\hline (14) payment for borrowing & 3.75 & 1.82 & 5.69 & .97 & 4.66 & 1.79 & 3.64 & 1.93 \\
\hline (13) hobby & 3.95 & 1.79 & 4.14 & .96 & 5.17 & 1.30 & 4.06 & 1.58 \\
\hline (12) concern & 3.51 & 1.81 & 4.42 & 1.09 & 5.03 & 1.21 & 3.69 & 1.78 \\
\hline INTRODUCTION & & & & & & & & \\
\hline (26) preamble & 3.95 & 1.87 & 4.83 & 1.04 & 4.86 & 1.74 & 3.17 & 1.83 \\
\hline (14) to meet & 4.46 & 1.88 & 4.31 & .75 & 5.29 & 1.36 & 4.08 & 1.88 \\
\hline IRON & & & & & & & & \\
\hline (24) for smoothing clothes & 6.05 & 1.39 & 3.06 & 1.17 & 5.80 & 1.41 & 6.11 & 1.39 \\
\hline $\begin{array}{l}\text { (16) metal } \\
\text { ISSUE }\end{array}$ & 4.95 & 1.74 & 3.75 & 1.04 & 5.60 & 1.18 & 5.75 & 1.72 \\
\hline (15) publication & 4.50 & 1.97 & 5.33 & 1.05 & 4.94 & 1.45 & 5.00 & 1.65 \\
\hline (14) that produced & 3.22 & 1.91 & 5.58 & 1.04 & 3.91 & 1.34 & 3.72 & 1.74 \\
\hline $\begin{array}{l}\text { (11) subject/topic } \\
\text { JACK }\end{array}$ & 2.89 & 1.69 & 5.33 & 1.03 & 4.20 & 1.31 & 3.14 & 1.73 \\
\hline (18) name & 3.16 & 1.95 & 2.64 & .92 & 4.31 & 1.74 & 4.53 & 2.08 \\
\hline (08) tool for lifting & 4.92 & 1.85 & 5.19 & 1.33 & 4.00 & 1.51 & 4.97 & 1.92 \\
\hline
\end{tabular}




\begin{tabular}{|c|c|c|c|c|c|c|c|c|}
\hline \multirow[b]{2}{*}{ Word and Meaning } & \multicolumn{2}{|c|}{ Imagery } & \multicolumn{2}{|c|}{ Age of Acquisition } & \multicolumn{2}{|c|}{ Familiarity } & \multicolumn{2}{|c|}{ Concreteness } \\
\hline & Mean & SD & Mean & SD & Mean & SD & Mean & SD \\
\hline $\begin{array}{l}\text { (06) honour in cards } \\
\text { JADE }\end{array}$ & 5.03 & 1.85 & 4.28 & 1.47 & 3.43 & 1.55 & 4.31 & 2.17 \\
\hline $\begin{array}{l}\text { (28) stone } \\
\text { (05) colour } \\
\text { JERSEY }\end{array}$ & $\begin{array}{l}5.28 \\
4.57\end{array}$ & $\begin{array}{l}1.59 \\
1.97\end{array}$ & $\begin{array}{l}5.34 \\
5.74\end{array}$ & $\begin{array}{l}.98 \\
.91\end{array}$ & $\begin{array}{l}4.51 \\
3.83\end{array}$ & $\begin{array}{l}1.76 \\
1.67\end{array}$ & $\begin{array}{l}5.53 \\
4.56\end{array}$ & $\begin{array}{l}1.82 \\
1.77\end{array}$ \\
\hline $\begin{array}{l}\text { (31) jumper } \\
\text { (06) island } \\
\text { JINGLE }\end{array}$ & $\begin{array}{l}6.00 \\
4.11\end{array}$ & $\begin{array}{l}1.33 \\
1.94\end{array}$ & $\begin{array}{l}3.17 \\
5.06\end{array}$ & $\begin{array}{l}1.30 \\
1.00\end{array}$ & $\begin{array}{l}5.49 \\
3.74\end{array}$ & $\begin{array}{l}1.48 \\
1.40\end{array}$ & $\begin{array}{l}6.03 \\
5.06\end{array}$ & $\begin{array}{l}1.34 \\
2.00\end{array}$ \\
\hline $\begin{array}{l}\text { (21) noise } \\
\text { (18) poem/song } \\
\text { JUNIOR }\end{array}$ & $\begin{array}{l}5.19 \\
4.24\end{array}$ & $\begin{array}{l}1.57 \\
1.97\end{array}$ & $\begin{array}{l}3.69 \\
5.43\end{array}$ & $\begin{array}{l}1.49 \\
1.34\end{array}$ & $\begin{array}{l}3.89 \\
3.97\end{array}$ & $\begin{array}{l}1.65 \\
1.63\end{array}$ & $\begin{array}{l}4.83 \\
4.36\end{array}$ & $\begin{array}{l}1.76 \\
2.02\end{array}$ \\
\hline $\begin{array}{l}\text { (22) low in status } \\
\text { (16) younger } \\
\text { KEEL }\end{array}$ & $\begin{array}{l}3.24 \\
3.70\end{array}$ & $\begin{array}{l}1.91 \\
1.96\end{array}$ & $\begin{array}{l}4.39 \\
4.46\end{array}$ & $\begin{array}{l}1.21 \\
1.16\end{array}$ & $\begin{array}{l}4.20 \\
4.23\end{array}$ & $\begin{array}{l}1.77 \\
1.57\end{array}$ & $\begin{array}{l}3.78 \\
4.22\end{array}$ & $\begin{array}{l}1.89 \\
1.90\end{array}$ \\
\hline $\begin{array}{l}\text { (27) part of boat } \\
\text { (11) fall over } \\
\text { LABOUR }\end{array}$ & $\begin{array}{l}4.78 \\
4.30\end{array}$ & $\begin{array}{l}1.73 \\
1.87\end{array}$ & $\begin{array}{l}5.31 \\
4.89\end{array}$ & $\begin{array}{l}1.43 \\
1.35\end{array}$ & $\begin{array}{l}4.37 \\
3.71\end{array}$ & $\begin{array}{l}1.76 \\
1.43\end{array}$ & $\begin{array}{l}5.36 \\
3.97\end{array}$ & $\begin{array}{l}1.95 \\
1.72\end{array}$ \\
\hline $\begin{array}{l}\text { (24) work } \\
\text { (15) political party } \\
\text { LAND }\end{array}$ & $\begin{array}{l}4.27 \\
4.62\end{array}$ & $\begin{array}{l}1.72 \\
2.01\end{array}$ & $\begin{array}{l}5.00 \\
5.49\end{array}$ & $\begin{array}{l}1.05 \\
1.13\end{array}$ & $\begin{array}{l}4.40 \\
5.43\end{array}$ & $\begin{array}{l}1.73 \\
1.34\end{array}$ & $\begin{array}{l}4.00 \\
4.47\end{array}$ & $\begin{array}{l}1.93 \\
1.94\end{array}$ \\
\hline $\begin{array}{l}\text { (30) area of ground } \\
\text { (06) come down from a height } \\
\text { (04) country } \\
\text { LAP } \\
\text { (21) knees }\end{array}$ & $\begin{array}{l}5.24 \\
4.67 \\
4.89 \\
\\
5.22\end{array}$ & $\begin{array}{l}1.58 \\
1.45 \\
1.90\end{array}$ & $\begin{array}{l}3.14 \\
3.19 \\
3.47\end{array}$ & $\begin{array}{r}.86 \\
1.00 \\
1.21 \\
\\
99\end{array}$ & $\begin{array}{l}5.54 \\
4.54 \\
5.03 \\
\\
4.51\end{array}$ & $\begin{array}{l}1.38 \\
1.27 \\
1.67 \\
1.57\end{array}$ & $\begin{array}{l}5.11 \\
3.67 \\
4.92 \\
5.36\end{array}$ & $\begin{array}{l}1.61 \\
1.73 \\
1.50 \\
\\
1.48\end{array}$ \\
\hline $\begin{array}{l}\text { (12) to drink } \\
\text { (05) circuit of race track } \\
\text { LAUNDRY }\end{array}$ & $\begin{array}{l}5.03 \\
4.87\end{array}$ & $\begin{array}{l}1.51 \\
1.57 \\
1.56\end{array}$ & $\begin{array}{l}3.64 \\
4.34 \\
4.36\end{array}$ & $\begin{array}{r}.99 \\
1.18 \\
.82\end{array}$ & $\begin{array}{l}4.11 \\
3.60 \\
4.26\end{array}$ & $\begin{array}{l}1.57 \\
1.76 \\
1.48\end{array}$ & $\begin{array}{l}5.36 \\
4.31 \\
4.97\end{array}$ & $\begin{array}{l}1.48 \\
1.91 \\
1.64\end{array}$ \\
\hline $\begin{array}{l}\text { (30) washing } \\
\text { (10) place to do washing } \\
\text { LEAD }\end{array}$ & $\begin{array}{l}5.76 \\
5.75\end{array}$ & $\begin{array}{l}1.42 \\
1.50\end{array}$ & $\begin{array}{l}3.94 \\
3.94\end{array}$ & $\begin{array}{l}1.25 \\
1.35\end{array}$ & $\begin{array}{l}5.29 \\
5.83\end{array}$ & $\begin{array}{l}1.39 \\
1.21\end{array}$ & $\begin{array}{l}6.03 \\
5.86\end{array}$ & $\begin{array}{l}1.34 \\
1.70\end{array}$ \\
\hline $\begin{array}{l}\text { (27) metal } \\
\text { (06) strap for animal } \\
\text { (05) to guide } \\
\text { LETTER }\end{array}$ & $\begin{array}{l}5.14 \\
5.41 \\
4.42\end{array}$ & $\begin{array}{l}1.62 \\
1.65 \\
1.91\end{array}$ & $\begin{array}{l}4.14 \\
3.20 \\
3.81\end{array}$ & $\begin{array}{r}.87 \\
1.06 \\
1.08\end{array}$ & $\begin{array}{l}5.20 \\
4.20 \\
4.74\end{array}$ & $\begin{array}{l}1.69 \\
1.56 \\
1.23\end{array}$ & $\begin{array}{l}5.28 \\
5.44 \\
4.50\end{array}$ & $\begin{array}{l}1.91 \\
1.86 \\
1.64\end{array}$ \\
\hline $\begin{array}{l}\text { (31) correspondence } \\
\text { (09) unit in alphabet } \\
\text { LIE }\end{array}$ & $\begin{array}{l}6.00 \\
5.08\end{array}$ & $\begin{array}{l}1.27 \\
1.82\end{array}$ & $\begin{array}{l}3.28 \\
2.64\end{array}$ & $\begin{array}{r}1.04 \\
.86\end{array}$ & $\begin{array}{l}6.17 \\
5.63\end{array}$ & $\begin{array}{l}1.25 \\
1.22\end{array}$ & $\begin{array}{l}6.00 \\
5.17\end{array}$ & $\begin{array}{l}1.25 \\
1.97\end{array}$ \\
\hline $\begin{array}{l}\text { (34) untruth } \\
\text { (06) horizontal position } \\
\text { LIFT }\end{array}$ & $\begin{array}{l}4.41 \\
5.37\end{array}$ & $\begin{array}{l}2.05 \\
1.48\end{array}$ & $\begin{array}{l}2.50 \\
2.77\end{array}$ & $\begin{array}{r}.73 \\
1.31\end{array}$ & $\begin{array}{l}5.89 \\
5.40\end{array}$ & $\begin{array}{l}1.09 \\
1.52\end{array}$ & $\begin{array}{l}3.75 \\
4.58\end{array}$ & $\begin{array}{l}2.02 \\
1.77\end{array}$ \\
\hline $\begin{array}{l}\text { (23) to pick up/raise } \\
\text { (16) elevator } \\
\text { LIME }\end{array}$ & $\begin{array}{l}4.65 \\
6.25\end{array}$ & $\begin{array}{l}1.71 \\
1.06\end{array}$ & $\begin{array}{l}3.00 \\
3.31\end{array}$ & $\begin{array}{l}1.29 \\
1.02\end{array}$ & $\begin{array}{l}5.69 \\
5.40\end{array}$ & $\begin{array}{l}1.47 \\
1.38\end{array}$ & $\begin{array}{l}4.81 \\
6.06\end{array}$ & $\begin{array}{l}1.66 \\
1.41\end{array}$ \\
\hline $\begin{array}{l}\text { (32) fruit/tree } \\
\text { (04) colour } \\
\text { (04) alkaline earth } \\
\text { LINE } \\
\text { (22) long narrow mark }\end{array}$ & $\begin{array}{l}5.06 \\
5.35 \\
3.56 \\
5.38\end{array}$ & $\begin{array}{l}1.83 \\
1.66 \\
1.94 \\
1.55\end{array}$ & $\begin{array}{l}4.58 \\
4.33 \\
6.28 \\
\\
2.69\end{array}$ & $\begin{array}{r}1.09 \\
1.11 \\
.90 \\
.91\end{array}$ & $\begin{array}{l}4.49 \\
3.94 \\
3.80 \\
6.06\end{array}$ & $\begin{array}{l}1.66 \\
1.53 \\
1.70 \\
1.12\end{array}$ & $\begin{array}{l}5.31 \\
4.53 \\
4.25 \\
\\
5.50\end{array}$ & $\begin{array}{l}1.85 \\
1.64 \\
1.94 \\
1.74\end{array}$ \\
\hline $\begin{array}{l}\text { (05) railway track } \\
\text { (05) long piece of twine } \\
\text { LIVER }\end{array}$ & $\begin{array}{l}5.40 \\
5.79 \\
4.78\end{array}$ & $\begin{array}{l}1.55 \\
1.52 \\
1.56\end{array}$ & $\begin{array}{l}2.09 \\
3.22 \\
4.06\end{array}$ & $\begin{array}{l}.91 \\
1.03 \\
1.45\end{array}$ & $\begin{array}{l}0.00 \\
4.66 \\
3.94\end{array}$ & $\begin{array}{l}1.12 \\
1.53 \\
1.88\end{array}$ & $\begin{array}{l}5.30 \\
5.36 \\
4.47\end{array}$ & $\begin{array}{l}1.14 \\
1.73 \\
2.09\end{array}$ \\
\hline $\begin{array}{l}\text { (27) part of body } \\
\text { (13) food } \\
\text { LOBBY }\end{array}$ & $\begin{array}{l}5.17 \\
6.60\end{array}$ & $\begin{array}{l}1.81 \\
1.41\end{array}$ & $\begin{array}{l}4.54 \\
3.56\end{array}$ & $\begin{array}{l}1.16 \\
1.07\end{array}$ & $\begin{array}{l}5.29 \\
5.26\end{array}$ & $\begin{array}{l}1.45 \\
1.48\end{array}$ & $\begin{array}{l}5.58 \\
5.61\end{array}$ & $\begin{array}{l}1.64 \\
1.78\end{array}$ \\
\hline $\begin{array}{l}\text { (27) entrance } \\
\text { (09) corridor } \\
\text { (04) seek influence }\end{array}$ & $\begin{array}{l}5.03 \\
4.73 \\
2.56\end{array}$ & $\begin{array}{l}1.54 \\
1.98 \\
1.74\end{array}$ & $\begin{array}{l}4.33 \\
4.19 \\
6.56\end{array}$ & $\begin{array}{r}1.60 \\
1.60 \\
.83\end{array}$ & $\begin{array}{l}4.49 \\
4.40 \\
2.60\end{array}$ & $\begin{array}{l}1.38 \\
1.76 \\
1.44\end{array}$ & $\begin{array}{l}5.28 \\
4.92 \\
2.81\end{array}$ & $\begin{array}{l}1.63 \\
1.88 \\
1.49\end{array}$ \\
\hline $\begin{array}{l}\text { LODGE } \\
\text { (28) house }\end{array}$ & 5.19 & 1.54 & 4.69 & 1.24 & 4.11 & 1.67 & 5.25 & 1.79 \\
\hline (04) to put/deposit & 3.42 & 1.80 & 5.60 & 1.05 & 3.51 & 1.25 & 3.19 & 1.47 \\
\hline $\begin{array}{l}\text { (04) to stay } \\
\text { LORD }\end{array}$ & 4.05 & 1.61 & 5.29 & .97 & 4.29 & 1.67 & 4.06 & 1.62 \\
\hline $\begin{array}{l}\text { (34) feudal superior } \\
\text { (05) God }\end{array}$ & $\begin{array}{l}4.97 \\
4.14\end{array}$ & $\begin{array}{l}1.77 \\
2.45\end{array}$ & $\begin{array}{l}4.53 \\
2.92\end{array}$ & $\begin{array}{l}1.40 \\
1.21\end{array}$ & $\begin{array}{l}4.31 \\
4.60\end{array}$ & $\begin{array}{l}1.77 \\
1.97\end{array}$ & $\begin{array}{l}4.67 \\
3.97\end{array}$ & $\begin{array}{l}1.80 \\
2.39\end{array}$ \\
\hline (27) wood/timber & 5.43 & 1.70 & 4.43 & 1.02 & 3.83 & 1.83 & 5.53 & 1.85 \\
\hline
\end{tabular}




\begin{tabular}{|c|c|c|c|c|c|c|c|c|}
\hline \multirow[b]{2}{*}{ Word and Meaning } & \multicolumn{2}{|c|}{ Imagery } & \multicolumn{2}{|c|}{ Age of Acquisition } & \multicolumn{2}{|c|}{ Familiarity } & \multicolumn{2}{|c|}{ Concreteness } \\
\hline & Mean & SD & Mean & SD & Mean & SD & Mean & SD \\
\hline (04) walk & 3.27 & 1.62 & 5.64 & 1.21 & 1.97 & 1.23 & 3.28 & 1.66 \\
\hline (04) weigh down & 3.56 & 1.80 & 5.22 & 1.13 & 3.31 & 1.47 & 3.25 & 1.57 \\
\hline MAIN & & & & & & & & \\
\hline (34) most important & 3.16 & 2.16 & 3.69 & 1.00 & 6.09 & 1.05 & 3.92 & 2.22 \\
\hline $\begin{array}{l}\text { (04) large supply of gas, water, etc. } \\
\text { MARBLE }\end{array}$ & 4.03 & 1.97 & 5.17 & 1.19 & 4.34 & 1.45 & 4.33 & 1.76 \\
\hline (24) stone & 5.97 & 1.31 & 4.53 & 1.19 & 4.71 & 1.67 & 5.67 & 1.68 \\
\hline $\begin{array}{l}\text { (15) glass ball } \\
\text { MARCH }\end{array}$ & 5.73 & 1.67 & 3.47 & 1.28 & 4.91 & 1.54 & 5.75 & 1.67 \\
\hline (27) month & 4.25 & 1.96 & 2.86 & .71 & 5.23 & 1.33 & 3.58 & 2.13 \\
\hline $\begin{array}{l}\text { (13) walk uniformly } \\
\text { MARGIN }\end{array}$ & 5.58 & 1.38 & 3.31 & .84 & 5.06 & 1.57 & 4.86 & 1.81 \\
\hline (32) space at edge of paper & 5.44 & 1.82 & 3.86 & 1.06 & 5.91 & 1.04 & 5.83 & 1.34 \\
\hline $\begin{array}{l}\text { (04) extra allowance } \\
\text { MARVEL }\end{array}$ & 2.83 & 1.59 & 5.67 & 1.00 & 3.71 & 1.54 & 3.03 & 1.42 \\
\hline (25) wonder & 4.11 & 1.83 & 4.36 & 1.08 & 4.17 & 1.48 & 3.75 & 2.03 \\
\hline (09) brand of dried milk & 5.72 & 1.54 & 5.36 & 1.34 & 4.54 & 1.61 & 5.25 & 2.01 \\
\hline $\begin{array}{l}\text { (05) name of magazine } \\
\text { MASS }\end{array}$ & 3.19 & 2.44 & 5.66 & 1.31 & 2.57 & 1.59 & 3.33 & 2.17 \\
\hline (18) crowd/large amount & 4.72 & 1.82 & 4.49 & 1.25 & 4.38 & 1.37 & 4.97 & 1.68 \\
\hline (14) eucharist & 2.58 & 1.98 & 5.40 & 1.71 & 3.09 & 1.98 & 3.83 & 2.17 \\
\hline $\begin{array}{l}\text { (08) weight/atomic density } \\
\text { MATERIAL }\end{array}$ & 3.30 & 1.75 & 6.49 & .73 & 4.77 & 1.79 & 3.89 & 2.04 \\
\hline (21) cloth & 5.47 & 1.52 & 3.42 & 1.09 & 5.68 & 1.05 & 5.94 & 1.24 \\
\hline $\begin{array}{l}\text { (16) substance } \\
\text { MATTER }\end{array}$ & 3.64 & 1.72 & 4.31 & 1.01 & 5.11 & 1.21 & 4.78 & 1.77 \\
\hline (23) material & 3.50 & 1.91 & 5.17 & 1.01 & 4.34 & 1.79 & 4.43 & 1.92 \\
\hline (07) subject & 2.75 & 1.55 & 4.89 & .99 & 4.00 & 1.43 & 3.75 & 1.79 \\
\hline $\begin{array}{l}\text { (07) something wrong } \\
\text { MEASURE }\end{array}$ & 3.11 & 2.01 & 2.92 & 1.12 & 5.40 & 1.40 & 3.89 & 2.08 \\
\hline (35) ascertain quantity & 3.73 & 1.95 & 3.94 & 1.09 & 5.69 & 1.26 & 4.14 & 1.87 \\
\hline $\begin{array}{l}\text { (05) precise amount } \\
\text { MIGHT }\end{array}$ & 3.78 & 1.82 & 4.50 & 1.19 & 4.71 & 1.36 & 3.83 & 1.74 \\
\hline (26) strength/power & 4.00 & 2.11 & 4.40 & 1.15 & 3.97 & 1.50 & 3.89 & 1.90 \\
\hline $\begin{array}{l}\text { (14) possibly } \\
\text { MINUTE }\end{array}$ & 2.57 & 1.93 & 3.11 & .82 & 6.00 & 1.10 & 3.06 & 2.03 \\
\hline (29) period of time & 4.31 & 2.15 & 2.71 & .66 & 6.57 & .80 & 4.28 & 2.50 \\
\hline $\begin{array}{l}\text { (11) very small } \\
\text { MISTRESS }\end{array}$ & 3.89 & 2.01 & 4.31 & 1.14 & 4.60 & 1.57 & 3.53 & 1.95 \\
\hline (26) female lover & 5.53 & 1.34 & 6.28 & .80 & 5.09 & 1.38 & 5.14 & 1.65 \\
\hline $\begin{array}{l}\text { (10) teacher/female superior } \\
\text { MORAL }\end{array}$ & 5.61 & 1.44 & 3.64 & 1.11 & 4.03 & 1.32 & 5.00 & 1.65 \\
\hline (31) ethic & 2.80 & 1.91 & 5.89 & 1.09 & 4.80 & 1.53 & 3.19 & 2.32 \\
\hline $\begin{array}{l}\text { (06) message in story } \\
\text { MORTAR }\end{array}$ & 3.57 & 2.11 & 5.03 & 1.28 & 4.74 & 1.44 & 3.39 & 2.14 \\
\hline (24) construction material & 4.06 & 2.12 & 5.28 & 1.02 & 3.83 & 1.75 & 5.25 & 2.07 \\
\hline (07) gun & 3.11 & 1.90 & 5.14 & 1.21 & 2.77 & 1.55 & 4.78 & 2.23 \\
\hline $\begin{array}{l}\text { (06) vessel for crushing } \\
\text { NAIL }\end{array}$ & 4.76 & 1.84 & 6.29 & .74 & 3.69 & 1.79 & 5.17 & 2.13 \\
\hline (29) small spike & 5.86 & 1.46 & 3.06 & .92 & 5.37 & 1.59 & 5.89 & 1.79 \\
\hline $\begin{array}{l}\text { (11) horny covering } \\
\text { NATURE }\end{array}$ & 4.73 & 1.93 & 3.50 & 1.68 & 4.37 & 1.81 & 4.89 & 2.05 \\
\hline (33) natural world & 5.00 & 1.86 & 3.74 & 1.13 & 5.57 & 1.42 & 4.97 & 1.88 \\
\hline $\begin{array}{l}\text { (07) type } \\
\text { NAVY }\end{array}$ & 3.05 & 1.85 & 5.00 & 1.10 & 4.40 & 1.53 & 2.50 & 1.50 \\
\hline (35) maritime army & 5.73 & 1.37 & 3.29 & .94 & 5.03 & 1.70 & 5.42 & 1.80 \\
\hline $\begin{array}{l}\text { (05) colour } \\
\text { NEEDLE }\end{array}$ & 5.65 & 1.66 & 3.34 & 1.19 & 4.94 & 1.76 & 4.81 & 1.79 \\
\hline (34) metal sewing rod & 6.08 & 1.36 & 2.94 & 1.08 & 5.89 & 1.19 & 6.22 & 1.36 \\
\hline $\begin{array}{l}\text { (06) to annoy } \\
\text { NERVE }\end{array}$ & 3.54 & 1.69 & 5.28 & 1.07 & 2.91 & 1.25 & 2.92 & 1.46 \\
\hline (28) neuron & 4.24 & 2.11 & 6.17 & .87 & 4.49 & 1.76 & 5.19 & 1.75 \\
\hline $\begin{array}{l}\text { (09) courage } \\
\text { NET }\end{array}$ & 4.36 & 1.57 & 4.64 & 1.06 & 4.83 & 1.25 & 3.53 & 1.74 \\
\hline (34) mesh & 5.73 & 1.33 & 3.50 & 1.09 & 5.06 & 1.41 & 5.36 & 1.60 \\
\hline (0S) complete/remainder & 2.17 & 1.36 & 6.06 & .91 & 3.14 & 1.50 & 2.36 & 1.23 \\
\hline
\end{tabular}




\begin{tabular}{|c|c|c|c|c|c|c|c|c|}
\hline \multirow[b]{2}{*}{ Word and Meaning } & \multicolumn{2}{|c|}{ Imagery } & \multicolumn{2}{|c|}{ Age of Acquisition } & \multicolumn{2}{|c|}{ Familiarity } & \multicolumn{2}{|c|}{ Concreteness } \\
\hline & Mean & SD & Mean & SD & Mean & SD & Mean & SD \\
\hline \multicolumn{9}{|l|}{ NIP } \\
\hline (30) pinch/bite & 5.14 & 1.63 & 3.28 & 1.35 & 4.63 & 1.46 & 5.61 & 1.44 \\
\hline (09) small drink & 4.92 & 1.36 & 5.89 & .95 & 4.00 & 1.67 & 5.11 & 1.69 \\
\hline \multicolumn{9}{|l|}{ NOTE } \\
\hline (19) message & 5.50 & 1.32 & 3.31 & .84 & 5.55 & 1.13 & 5.71 & 1.14 \\
\hline (16) music & 5.08 & 1.55 & 3.72 & 1.17 & 4.66 & 1.55 & 4.89 & 1.90 \\
\hline (04) heed/jot down & 4.06 & 2.05 & 4.69 & .94 & 5.46 & 1.34 & 3.92 & 1.67 \\
\hline \multicolumn{9}{|l|}{ NOTICE } \\
\hline (22) sign/poster & 5.62 & 1.48 & 3.56 & 1.09 & 5.43 & 1.20 & 5.53 & 1.48 \\
\hline (18) heed & 3.00 & 1.54 & 3.92 & 1.21 & 5.06 & 1.22 & 3.61 & 1.53 \\
\hline \multicolumn{9}{|l|}{ NOVEL } \\
\hline (35) book & 5.81 & 1.45 & 4.42 & 1.14 & 5.89 & 1.35 & 5.81 & $\begin{array}{l}1.63 \\
158\end{array}$ \\
\hline (05) new & 3.00 & 1.64 & 5.44 & 1.12 & 3.83 & 1.32 & 2.89 & 1.58 \\
\hline \multicolumn{9}{|l|}{ OBJECT } \\
\hline (25) material thing & 3.97 & 2.12 & 3.94 & 1.13 & 5.83 & 1.23 & 5.60 & 1.61 \\
\hline (10) oppose & 3.42 & 1.82 & 4.78 & .82 & 4.80 & 1.41 & 3.11 & 1.61 \\
\hline \multicolumn{9}{|l|}{ OBSERVER } \\
\hline (30) one who watches & 5.03 & 1.76 & 4.92 & 1.06 & 5.46 & 1.25 & 4.81 & 1.78 \\
\hline (10) newspaper & 5.83 & 1.40 & 5.39 & 1.23 & 4.49 & 1.66 & 5.28 & 2.04 \\
\hline \multicolumn{9}{|l|}{ OPENING } \\
\hline (20) space & 4.30 & 1.71 & 3.35 & 1.03 & 5.06 & 1.37 & 4.22 & 1.73 \\
\hline (14) entrance & 5.03 & 1.70 & 3.09 & 1.11 & 5.20 & 1.35 & 4.86 & 1.40 \\
\hline (04) beginning & 3.28 & 1.74 & 4.29 & 1.23 & 4.11 & 1.51 & 3.36 & 1.73 \\
\hline \multicolumn{9}{|l|}{ ORANGE } \\
\hline $\begin{array}{l}\text { (33) fruit } \\
\text { (06) colour }\end{array}$ & $\begin{array}{l}6.43 \\
6.24\end{array}$ & $\begin{array}{l}1.00 \\
1.28\end{array}$ & $\begin{array}{l}2.26 \\
2.34\end{array}$ & $\begin{array}{l}.87 \\
.86\end{array}$ & $\begin{array}{l}6.31 \\
5.60\end{array}$ & $\begin{array}{r}.85 \\
1.07\end{array}$ & $\begin{array}{l}6.44 \\
5.31\end{array}$ & $\begin{array}{l}1.14 \\
1.65\end{array}$ \\
\hline $\begin{array}{l}\text { ORDER } \\
\text { ORDEIT }\end{array}$ & & & & & & & & \\
\hline (19) command & 4.68 & 1.68 & 3.47 & 1.04 & 5.46 & 1.36 & 4.44 & 1.62 \\
\hline (17) arrangement & 3.22 & 1.65 & 4.11 & 1.26 & 5.00 & 1.35 & 3.08 & 1.42 \\
\hline ORGAN & & & & & & & & \\
\hline (30) musical instrument & 6.03 & 1.28 & 3.61 & 1.09 & 4.89 & 1.82 & $\begin{array}{l}5.64 \\
5.20\end{array}$ & $\begin{array}{l}1.63 \\
159\end{array}$ \\
\hline (09) part of body & 4.76 & 1.85 & 5.46 & 1.16 & 5.14 & 1.44 & 5.39 & 1.59 \\
\hline $\begin{array}{l}\text { OVERTONE } \\
\text { (10) sound }\end{array}$ & 3.03 & 1.95 & 5.86 & .92 & 3.66 & 1.55 & 3.89 & 1.82 \\
\hline $\begin{array}{l}\text { (10) sound } \\
\text { (07) hint }\end{array}$ & 2.81 & 1.91 & 6.26 & .81 & 3.46 & 1.78 & $\begin{array}{l}3.09 \\
2.63\end{array}$ & $\begin{array}{l}1.02 \\
1.49\end{array}$ \\
\hline $\begin{array}{l}\text { (07) hint } \\
\text { (07) insinuation }\end{array}$ & 3.00 & 1.86 & 6.14 & 1.06 & 3.50 & 1.56 & 2.97 & 1.69 \\
\hline PALETTE & & & & & & & & \\
\hline (28) painting equipment & 5.62 & 1.44 & 4.83 & 1.24 & 3.91 & 1.84 & 5.61 & 1.69 \\
\hline (08) roof of mouth & 4.75 & 1.95 & 5.39 & 1.34 & 3.80 & 1.85 & 5.42 & 1.94 \\
\hline PALM & & & & & & & & \\
\hline (28) part of hand & 5.94 & 1.15 & 3.26 & .87 & 5.66 & 1.19 & $\begin{array}{l}6.36 \\
5.97\end{array}$ & $\begin{array}{l}1.00 \\
1.40\end{array}$ \\
\hline (12) tree & 6.08 & 1.08 & 3.86 & 1.11 & 4.51 & 1.63 & 5.97 & 1.40 \\
\hline $\begin{array}{l}\text { PARDON } \\
\text { (20) apology/excuse }\end{array}$ & 3.46 & 1.95 & & & 5.40 & 1.36 & 3.60 & 1.81 \\
\hline $\begin{array}{l}\text { (20) apology/excuse } \\
\text { (20) remission }\end{array}$ & 3.62 & 1.82 & $\begin{array}{l}3.20 \\
4.64\end{array}$ & 1.36 & 3.86 & 1.44 & 3.64 & 1.78 \\
\hline $\begin{array}{l}\text { (20) remission } \\
\text { PARK }\end{array}$ & & & & & & & & \\
\hline (32) leisure area & 5.73 & 1.27 & 2.58 & .83 & 5.46 & 1.57 & 5.67 & 1.08 \\
\hline (06) leave vehicle stationary & 5.14 & 1.36 & 3.22 & .82 & 5.60 & 1.48 & 4.28 & 1.90 \\
\hline $\begin{array}{l}\text { (32) section } \\
(07) \text { to separate }\end{array}$ & $\begin{array}{l}4.11 \\
3.64\end{array}$ & $\begin{array}{l}1.96 \\
1.77\end{array}$ & 4.19 & 1.13 & 4.89 & 1.56 & $\begin{array}{l}4.90 \\
3.97\end{array}$ & $\begin{array}{l}1.00 \\
1.55\end{array}$ \\
\hline $\begin{array}{l}\text { (07) to separate } \\
\text { PASSAGE }\end{array}$ & 3.64 & & & & & & & \\
\hline (24) corridor & 5.57 & 1.57 & 3.78 & 1.03 & 5.23 & 1.20 & 5.58 & 1.30 \\
\hline (10) journey/route & 3.69 & 1.60 & 4.81 & .88 & 3.20 & 1.26 & 4.03 & 1.69 \\
\hline (04) part of text & 4.32 & 1.43 & 4.83 & .96 & 4.71 & 1.50 & 4.03 & 1.76 \\
\hline PASTE & & & & & & & & \\
\hline (27) glue & 5.78 & 1.21 & 3.09 & .77 & 4.57 & 1.66 & 5.75 & 1.44 \\
\hline (09) soft solid & 4.67 & 1.73 & 3.69 & 1.17 & 4.31 & 1.39 & 5.36 & 1.48 \\
\hline (04) food & 4.08 & 1.73 & 4.85 & 1.33 & 3.06 & 1.57 & 4.56 & 2.06 \\
\hline PATIENCE & & & & & & & & \\
\hline (32) tolerance & 3.51 & 1.90 & 4.29 & 1.19 & 5.69 & 1.12 & $\begin{array}{r}3.64 \\
152\end{array}$ & 2.12 \\
\hline $\begin{array}{l}\text { (07) card game } \\
\text { PATIENT }\end{array}$ & 5.14 & 1.85 & 4.09 & 1.23 & $4.00^{\circ}$ & 1.31 & 4.53 & 1.80 \\
\hline $\begin{array}{l}\text { PATIENT } \\
\text { (31) person ill }\end{array}$ & 5.73 & 1.48 & 3.39 & 1.04 & 5.46 & 1.27 & 5.81 & 1.31 \\
\hline (08) tolerant & 3.89 & 1.90 & 4.09 & 1.08 & 5.26 & 1.08 & 3.46 & 1.89 \\
\hline $\begin{array}{l}\text { PECK } \\
\text { (29) strike with a beak }\end{array}$ & 5.32 & 1.23 & 3.34 & 1.17 & 4.89 & 1.47 & 5.08 & 1.71 \\
\hline (29) strike with a beak & 0.02 & & & & & & & \\
\hline
\end{tabular}




\begin{tabular}{|c|c|c|c|c|c|c|c|c|}
\hline \multirow[b]{2}{*}{ Word and Meaning } & \multicolumn{2}{|c|}{ Imagery } & \multicolumn{2}{|c|}{ Age of Acquisition } & \multicolumn{2}{|c|}{ Familiarity } & \multicolumn{2}{|c|}{ Concreteness } \\
\hline & Mean & SD & Mean & SD & Mean & SD & Mean & SD \\
\hline $\begin{array}{l}\text { (09) nibble } \\
\text { PEEP }\end{array}$ & 4.92 & 1.52 & 3.56 & 1.38 & 4.20 & 1.55 & 4.50 & 1.40 \\
\hline (28) look at & 5.08 & 1.59 & 2.83 & 1.07 & 5.11 & 1.37 & 4.78 & 1.62 \\
\hline $\begin{array}{l}\text { (11) noise } \\
\text { PELT }\end{array}$ & 4.00 & 1.86 & 3.28 & 1.39 & 3.46 & 1.56 & 3.94 & 1.99 \\
\hline (18) skin & 4.97 & 1.72 & 5.46 & 1.44 & 3.17 & 1.70 & 4.53 & 2.02 \\
\hline (17) hit hard & 4.24 & 1.82 & 4.36 & 1.38 & 3.66 & 1.49 & 3.83 & 1.79 \\
\hline $\begin{array}{l}\text { (04) rain heavily } \\
\text { PERCH }\end{array}$ & 5.35 & 1.68 & 4.25 & 1.26 & 4.77 & 1.29 & 5.00 & 1.78 \\
\hline (30) object on which to stand & 4.78 & 1.47 & 4.37 & 1.27 & 3.97 & 1.58 & 5.03 & 1.85 \\
\hline $\begin{array}{l}(08) \text { fish } \\
\text { PERIOD }\end{array}$ & 4.32 & 1.96 & 5.17 & 1.13 & 2.91 & 1.44 & 4.72 & 2.35 \\
\hline (31) length of time & 3.92 & 2.01 & 4.50 & 1.09 & 5.41 & 1.24 & 4.11 & 1.92 \\
\hline $\begin{array}{l}\text { (08) menstruation } \\
\text { PERMIT }\end{array}$ & 5.26 & 1.73 & 5.63 & 1.17 & 5.29 & 1.45 & 5.42 & 1.75 \\
\hline (26) allow & 3.43 & 1.94 & 4.56 & 1.28 & 5.37 & 1.57 & 4.03 & 2.05 \\
\hline $\begin{array}{l}\text { (14) document } \\
\text { PEST }\end{array}$ & 4.38 & 1.81 & 5.33 & .97 & 4.09 & 1.44 & 4.81 & 1.52 \\
\hline (33) undesireable & 4.78 & 1.86 & 3.46 & 1.27 & 5.20 & 1.26 & 4.36 & 1.81 \\
\hline $\begin{array}{l}\text { (06) insect } \\
\text { PIPING }\end{array}$ & 5.39 & 1.50 & 4.11 & 1.29 & 3.89 & 1.51 & 5.47 & 1.44 \\
\hline (14) metal tubing & 5.14 & 1.55 & 4.50 & 1.24 & 4.29 & 1.52 & 5.58 & 1.55 \\
\hline (11) playing musical instrument & 4.44 & 1.71 & 4.28 & 1.22 & 3.35 & 1.26 & 4.60 & 1.78 \\
\hline (05) very hot & 4.78 & 1.80 & 4.53 & 1.32 & 4.14 & 1.52 & 3.61 & 1.82 \\
\hline $\begin{array}{l}\text { (05) stitching } \\
\text { PLACE }\end{array}$ & 3.70 & 2.26 & 6.06 & 1.15 & 2.17 & 1.56 & 3.58 & 2.02 \\
\hline (32) locality & 4.19 & 2.01 & 3.39 & 1.25 & 5.89 & 1.19 & 4.47 & 1.80 \\
\hline $\begin{array}{l}\text { (07) to put } \\
\text { PLAIN }\end{array}$ & 3.81 & 1.89 & 3.51 & 1.25 & 4.86 & 1.42 & 3.97 & 1.59 \\
\hline (28) ordinary & 3.72 & 2.16 & 3.67 & 1.11 & 5.43 & 1.20 & 3.97 & 2.05 \\
\hline $\begin{array}{l}\text { (08) flat land } \\
\text { PLAY }\end{array}$ & 4.92 & 1.55 & 4.58 & .92 & 3.51 & 1.23 & 4.64 & 1.84 \\
\hline (21) dramatic piece & 5.11 & 1.33 & 4.25 & 1.12 & 5.26 & 1.50 & 4.67 & 1.65 \\
\hline (12) general recreation & 4.47 & 1.72 & 2.28 & 1.10 & 4.88 & 1.49 & 4.49 & 1.70 \\
\hline $\begin{array}{l}\text { (04) partake of game } \\
\text { PLOT }\end{array}$ & 4.44 & 1.76 & 2.17 & .87 & 5.63 & 1.31 & 4.50 & 1.36 \\
\hline (27) plan against & 4.36 & 1.80 & 4.14 & 1.18 & 5.14 & 1.38 & 4.06 & 1.87 \\
\hline (08) essence of story & 4.33 & 1.67 & 4.64 & 1.16 & 4.60 & 1.31 & 3.58 & 1.89 \\
\hline $\begin{array}{l}\text { (04) piece of land } \\
\text { PLUG }\end{array}$ & 4.97 & 1.52 & 4.22 & 1.08 & 4.18 & 1.36 & 4.78 & 1.80 \\
\hline (25) stopper & 5.46 & 1.48 & 3.11 & 1.15 & 5.14 & 1.46 & 5.75 & 1.53 \\
\hline $\begin{array}{l}\text { (14) electrical connector } \\
\text { PORT }\end{array}$ & 6.05 & 1.39 & 3.53 & 1.32 & 6.11 & 1.33 & 6.00 & 1.60 \\
\hline (26) dock/harbour & 6.16 & 1.13 & 3.42 & .95 & 5.17 & 1.50 & 6.17 & 1.26 \\
\hline $\begin{array}{l}\text { (12) wine } \\
\text { POSITION }\end{array}$ & 5.57 & 1.53 & 5.50 & 1.01 & 4.54 & 1.44 & 5.56 & 1.76 \\
\hline (24) place/point & 3.54 & 1.81 & 3.91 & 1.05 & 5.03 & 1.63 & 4.67 & 1.65 \\
\hline (09) status & 3.75 & 2.07 & 4.89 & 1.07 & 5.06 & 1.41 & 3.64 & 1.86 \\
\hline $\begin{array}{l}(04) \text { orientation } \\
\text { POST }\end{array}$ & 3.31 & 1.85 & 4.58 & 1.28 & 5.00 & 1.72 & 3.22 & 1.77 \\
\hline (31) send by mail & 5.54 & 1.65 & 3.28 & .93 & 6.37 & .83 & 5.33 & 1.72 \\
\hline $\begin{array}{l}\text { (07) stick of wood } \\
\text { POT }\end{array}$ & 5.14 & 1.70 & 3.47 & 1.17 & 4.37 & 1.17 & 5.08 & 2.01 \\
\hline (25) cooking u tensil & 5.89 & 1.47 & 2.63 & 1.02 & 5.71 & 1.30 & 5.97 & 1.42 \\
\hline (08) drug & 4.72 & 1.98 & 6.50 & .69 & 4.26 & 1.75 & 4.81 & 2.26 \\
\hline $\begin{array}{l}\text { (05) plant container } \\
\text { POUND }\end{array}$ & 5.22 & 1.70 & 3.00 & .97 & 5.38 & 1.21 & 5.83 & 1.63 \\
\hline (32) money & 6.30 & 1.04 & 3.08 & 1.12 & 6.46 & 1.00 & 5.86 & 1.77 \\
\hline $\begin{array}{l}\text { (06) weight } \\
\text { PRACTICAL }\end{array}$ & 3.95 & 1.96 & 3.60 & .87 & 5.43 & 1.42 & 4.39 & 1.78 \\
\hline (13) useful & 3.19 & 2.00 & 4.66 & 1.04 & 5.43 & 1.25 & 3.67 & 2.16 \\
\hline (11) lab-class & 5.89 & 1.35 & 6.42 & .80 & 5.31 & 1.79 & 5.22 & 1.78 \\
\hline (08) applied/down to earth & 3.57 & 1.75 & 5.26 & 1.00 & 5.37 & 1.24 & 3.61 & 1.83 \\
\hline $\begin{array}{l}\text { (07) having manual skill } \\
\text { PRELUDE }\end{array}$ & 3.46 & 1.64 & 5.14 & 1.00 & 4.79 & 1.71 & 3.72 & 1.61 \\
\hline (26) introduction & 3.70 & 1.67 & 5.69 & 1.10 & 4.51 & 1.57 & 3.92 & 2.01 \\
\hline (12) musical introduction & 4.24 & 1.62 & 6.00 & 1.12 & 4.03 & 1.72 & 4.36 & 2.04 \\
\hline
\end{tabular}




\begin{tabular}{|c|c|c|c|c|c|c|c|c|}
\hline \multirow[b]{2}{*}{ Word and Meaning } & \multicolumn{2}{|c|}{ Imagery } & \multicolumn{2}{|c|}{ Age of Acquisition } & \multicolumn{2}{|c|}{ Familiarity } & \multicolumn{2}{|c|}{ Concreteness } \\
\hline & Mean & SD & Mean & SD & Mean & SD & Mean & SD \\
\hline \multicolumn{9}{|l|}{ PRESSURE } \\
\hline (36) measure of force & 4.11 & 2.09 & 5.53 & 1.09 & 5.09 & 1.13 & 4.08 & 1.82 \\
\hline (04) emotional stress & 4.76 & 1.72 & 5.67 & 1.00 & 5.20 & 1.14 & 3.67 & 1.93 \\
\hline \multicolumn{9}{|l|}{ PRIMARY } \\
\hline $\begin{array}{l}\text { (21) first } \\
\text { (15) eariy school }\end{array}$ & 3.16 & 1.85 & 4.36 & 1.42 & 4.80 & 1.35 & 3.89 & 2.12 \\
\hline $\begin{array}{l}\text { (15) early school } \\
\text { (04) basic }\end{array}$ & 5.27 & 1.84 & 3.06 & 1.33 & 5.20 & 1.43 & 4.94 & 1.87 \\
\hline $\begin{array}{l}\text { (04) basic } \\
\text { PRINCIPAL }\end{array}$ & 2.61 & 1.55 & 4.86 & 1.27 & 4.06 & 1.55 & 3.28 & 1.80 \\
\hline \multicolumn{9}{|l|}{ PRINCIPAL } \\
\hline $\begin{array}{l}\text { (29) head of institution } \\
\text { (09) main }\end{array}$ & 4.19 & 1.91 & 5.08 & 1.23 & 4.89 & 1.41 & 5.25 & 1.67 \\
\hline $\begin{array}{l}\text { (09) main } \\
\text { PRINCIPLE }\end{array}$ & 2.60 & 1.62 & 4.78 & 1.00 & 5.03 & 1.52 & 3.66 & 2.04 \\
\hline \multicolumn{9}{|l|}{ PRINCIPLE } \\
\hline (22) belief/fundamental truth & 3.05 & 2.08 & 5.83 & .81 & 4.86 & 1.68 & 3.39 & 2.15 \\
\hline (09) head of & 3.95 & 1.97 & 5.06 & 1.25 & 4.09 & 1.78 & 4.42 & 1.86 \\
\hline (07) main & 2.65 & 1.77 & 5.11 & 1.05 & 4.77 & 1.76 & 3.83 & 2.08 \\
\hline \multicolumn{9}{|l|}{ PROBLEM } \\
\hline (20) question & 3.89 & 2.04 & 3.92 & .86 & 5.23 & 1.35 & 3.69 & 1.66 \\
\hline (14) difficult action & 3.22 & 1.96 & 4.29 & 1.14 & 4.77 & 1.74 & 3.75 & 1.72 \\
\hline (06) uncertainty & 3.32 & 1.68 & 3.94 & 1.04 & 4.86 & 1.52 & 3.56 & 1.79 \\
\hline \multicolumn{9}{|l|}{ PRODUCE } \\
\hline (32) goods made or grown & 4.68 & 1.77 & 4.72 & .90 & 5.09 & 1.32 & 4.72 & 1.76 \\
\hline (05) present/bring forward & 3.58 & 1.96 & 4.71 & 1.16 & 4.09 & 1.54 & 3.25 & 1.52 \\
\hline \multicolumn{3}{|l|}{ PRODUCTION } & 5.39 & .76 & 4.14 & & & \\
\hline $\begin{array}{l}\text { (30) manufacture } \\
\text { (09) a play }\end{array}$ & 4.51 & 1.67 & 4.83 & .90 & 4.60 & 1.48 & $\begin{array}{l}3.94 \\
4.44\end{array}$ & $\begin{array}{l}1.02 \\
1.72\end{array}$ \\
\hline $\begin{array}{l}\text { (09) a play } \\
\text { PROGRAMME }\end{array}$ & & & & & & & & \\
\hline (19) television broadcast & 5.97 & 1.26 & 3.39 & .95 & 6.26 & .84 & 5.61 & 1.66 \\
\hline (17) agenda/sequence of proceedings & 3.78 & 1.83 & 5.11 & 1.14 & 4.80 & 1.21 & 3.69 & 1.79 \\
\hline PROMENADE & & & & & & & & \\
\hline (32) place for walking & $\begin{array}{l}5.75 \\
4.41\end{array}$ & 1.61 & 5.14 & 1.03 & 4.80 & 1.64 & 5.78 & 1.06 \\
\hline (08) to walk & 4.41 & 1.73 & 5.22 & 1.42 & 3.17 & 1.75 & 4.08 & 1.92 \\
\hline PROVISION & & & & & & & & \\
\hline (19) preparation & 2.97 & 1.64 & 5.57 & 1.10 & 3.94 & 1.37 & 3.11 & 1.51 \\
\hline (18) stock of food & 4.53 & 1.72 & 5.11 & 1.17 & .4 .09 & 1.40 & 4.56 & 1.85 \\
\hline PRUNE & & & & & & & & \\
\hline (31) dried fruit & 5.94 & 1.15 & 3.58 & 1.19 & 5.14 & 1.76 & 6.28 & .96 \\
\hline (09) to cut plants & 4.62 & 1.65 & 4.75 & 1.19 & 4.29 & 1.65 & 4.31 & 1.82 \\
\hline PUNCH & & & & & & & & \\
\hline (29) hit & 5.84 & 1.39 & 2.83 & .99 & 5.56 & 1.27 & 5.25 & 1.42 \\
\hline (04) drink & 5.69 & 1.49 & 5.60 & 1.02 & 4.31 & 1.51 & 5.28 & 1.61 \\
\hline (04) magazine & 5.87 & 1.36 & 5.69 & 1.29 & 4.06 & 1.62 & 4.89 & 1.87 \\
\hline QUIVER & & & & & & & & \\
\hline (22) shake & 5.05 & 1.18 & 4.67 & 1.13 & 4.46 & 1.32 & 5.00 & 1.51 \\
\hline $\begin{array}{l}\text { (18) container for arrows } \\
\text { RACKET }\end{array}$ & 5.08 & 1.70 & 4.71 & 1.23 & 3.31 & 1.70 & 4.94 & 2.11 \\
\hline (24) sporting equipment & 6.30 & .90 & 3.97 & 1.30 & 5.49 & 1.38 & 6.19 & 1.41 \\
\hline (13) loud noise & 4.87 & 1.82 & 3.44 & .96 & 5.26 & 1.13 & 4.50 & 1.86 \\
\hline RANGE & & & & & & & & \\
\hline (12) distance reachable & 3.38 & 1.70 & 5.08 & 1.23 & 4.06 & 1.31 & 4.03 & 1.69 \\
\hline (11) variety/scope & 3.00 & 1.70 & 5.08 & .98 & 5.26 & 1.38 & 3.71 & 1.88 \\
\hline (08) area of land & 3.84 & 1.53 & 4.69 & .98 & 3.34 & 1.33 & 4.11 & 1.68 \\
\hline $\begin{array}{l}\text { (08) area for shooting } \\
\text { RATE }\end{array}$ & 4.83 & 1.69 & 5.33 & 1.11 & 4.00 & 1.55 & 4.92 & 1.79 \\
\hline (27) speed/degree of change & 2.73 & 1.61 & 5.39 & 1.06 & 5.17 & 1.36 & 3.42 & 2.11 \\
\hline (05) to grade & 2.83 & 1.34 & 5.50 & .90 & 4.46 & 1.61 & 3.67 & 1.86 \\
\hline RATTLE & & & & & & & & \\
\hline (20) toy & 5.41 & 1.53 & 1.89 & .79 & 4.37 & 1.74 & 5.83 & 1.52 \\
\hline (10) noise & 5.24 & 1.55 & 2.67 & 1.13 & 4.97 & 1.44 & 4.75 & 1.61 \\
\hline (07) to shake & 5.03 & 1.54 & 2.81 & 1.29 & 4.54 & 1.68 & 5.06 & 1.63 \\
\hline RAY & & & & & & & & \\
\hline (33) beam of light & 5.81 & 1.37 & 4.14 & 1.32 & 4.83 & 1.36 & 5.28 & 1.77 \\
\hline (04) fish & 3.89 & 2.02 & 5.29 & 1.30 & 2.60 & 1.34 & 4.22 & 2.38 \\
\hline REACTION & & & & & & & & \\
\hline (32) action in response & 4.35 & 1.68 & 4.86 & .95 & 5.71 & 1.06 & 4.58 & 1.82 \\
\hline (07) chemical change & 4.38 & 1.71 & 6.14 & .71 & 5.12 & 1.49 & 4.11 & 1.91 \\
\hline REALM & & & & & & & & \\
\hline (21) kingdom & 4.22 & 1.77 & 4.29 & 1.23 & 4.26 & 1.81 & 4.11 & 1.82 \\
\hline (10) sphere/domain & 3.24 & 2.03 & 4.97 & 1.34 & 3.91 & 1.75 & 3.36 & 1.99 \\
\hline
\end{tabular}




\begin{tabular}{|c|c|c|c|c|c|c|c|c|}
\hline \multirow[b]{2}{*}{ Word and Meaning } & \multicolumn{2}{|c|}{ Imagery } & \multicolumn{2}{|c|}{ Age of Acquisition } & \multicolumn{2}{|c|}{ Familiarity } & \multicolumn{2}{|c|}{ Concreteness } \\
\hline & Mean & SD & Mean & SD & Mean & SD & Mean & SD \\
\hline \multicolumn{9}{|l|}{ REASON } \\
\hline (14) excuse & 2.92 & 1.53 & 3.94 & 1.00 & 5.37 & 1.48 & 3.47 & 1.72 \\
\hline (11) explanation & 3.41 & 1.78 & 3.78 & 1.23 & 5.91 & 1.05 & 4.00 & 2.03 \\
\hline (08) purpose & 3.00 & 1.80 & 3.97 & 1.04 & 5.63 & 1.35 & 3.36 & 2.04 \\
\hline $\begin{array}{l}\text { (06) thought process } \\
\text { RECORD }\end{array}$ & 3.39 & 1.93 & 5.20 & 1.06 & 5.23 & 1.48 & 3.50 & 1.92 \\
\hline (32) preserving of sounds & 5.32 & 1.43 & 4.67 & 1.41 & 5.86 & 1.25 & 4.64 & 2.06 \\
\hline $\begin{array}{l}\text { (06) take note of } \\
\text { REED }\end{array}$ & 4.30 & 1.54 & 4.74 & 1.10 & 4.65 & 1.53 & 3.61 & 1.77 \\
\hline (31) plant & 5.81 & 1.52 & 3.94 & 1.22 & 4.26 & 1.54 & 5.97 & 1.42 \\
\hline $\begin{array}{l}\text { (06) part of musical instrument } \\
\text { REFORMATION }\end{array}$ & 5.11 & 1.94 & 5.64 & 1.03 & 3.23 & 1.51 & 5.06 & 2.04 \\
\hline (26) change & 2.81 & 1.69 & 5.94 & .97 & 3.77 & 1.65 & 3.29 & 1.80 \\
\hline $\begin{array}{l}\text { (12) period of religious change } \\
\text { REFUSE }\end{array}$ & 3.27 & 1.90 & 6.34 & .86 & 3.94 & 2.07 & 3.75 & 2.02 \\
\hline (20) decline & 3.87 & 1.99 & 3.97 & 1.21 & 5.46 & 1.40 & 4.06 & 1.89 \\
\hline (20) waste & 5.16 & 1.55 & 4.67 & 1.23 & 4.51 & 1.52 & 5.08 & 1.52 \\
\hline \multicolumn{9}{|l|}{ REGISTER } \\
\hline $\begin{array}{l}\text { (08) include a name } \\
\text { (0) }\end{array}$ & $\begin{array}{l}4.89 \\
2.81\end{array}$ & $\begin{array}{l}1.60 \\
1.52\end{array}$ & $\begin{array}{l}4.26 \\
5.06\end{array}$ & $\begin{array}{l}1.30 \\
1.33\end{array}$ & $\begin{array}{l}5.29 \\
3.94\end{array}$ & $\begin{array}{l}1.34 \\
1.31\end{array}$ & $\begin{array}{l}5.36 \\
3.58\end{array}$ & $\begin{array}{l}1.42 \\
1.74\end{array}$ \\
\hline (08) written record & 4.67 & 1.73 & 4.53 & 1.28 & 5.20 & 1.24 & 5.06 & 1.93 \\
\hline $\begin{array}{l}\text { (04) cash till } \\
\text { RELATION }\end{array}$ & 5.00 & 1.56 & 5.17 & .97 & 4.11 & 1.51 & 5.17 & 1.95 \\
\hline (36) person of kin & 6.00 & 1.27 & 3.50 & .99 & 6.31 & .82 & 5.78 & 1.51 \\
\hline $\begin{array}{l}\text { (04) link } \\
\text { REMEDY }\end{array}$ & 3.05 & 1.59 & 4.94 & .97 & 4.03 & 1.28 & 3.06 & 1.45 \\
\hline (35) panacea/cure & 4.08 & 1.82 & 4.71 & 1.08 & 5.31 & 1.37 & 4.14 & 1.86 \\
\hline $\begin{array}{l}\text { (05) solution } \\
\text { REPORT }\end{array}$ & 3.22 & 2.04 & 5.20 & 1.06 & 4.57 & 1.70 & 3.50 & 1.94 \\
\hline (26) account of & 4.08 & 1.77 & 4.69 & 1.20 & 5.71 & 1.06 & 4.19 & 1.70 \\
\hline (07) inform/complain & 3.84 & 1.72 & 4.17 & .90 & 4.83 & 1.34 & 3.47 & 1.76 \\
\hline $\begin{array}{l}\text { (05) bang from gun } \\
\text { RESISTANCE }\end{array}$ & 3.64 & 2.11 & 5.61 & 1.21 & 2.57 & 1.55 & 3.36 & 2.04 \\
\hline (25) opposition & 3.70 & 1.94 & 5.20 & .95 & 4.86 & 1.44 & 3.60 & 1.95 \\
\hline $\begin{array}{l}\text { (09) opposition to electric current } \\
\text { REST }\end{array}$ & 2.81 & 1.81 & 6.53 & .76 & 3.29 & 1.45 & 3.39 & 1.95 \\
\hline (26) repose & 4.32 & 1.89 & 2.94 & 1.35 & 5.46 & 1.46 & 4.67 & 1.60 \\
\hline (09) stop action & 4.44 & 1.77 & 2.83 & .93 & 5.71 & 1.34 & 4.14 & 1.86 \\
\hline $\begin{array}{l}\text { (05) remainder } \\
\text { REVIEW }\end{array}$ & \multicolumn{7}{|c|}{ REVIEW } & 1.70 \\
\hline (22) survey & 3.11 & 1.74 & 5.60 & .84 & 4.14 & 1.40 & 3.97 & 1.62 \\
\hline $\begin{array}{l}\text { (16) critical notice } \\
\text { REVOLUTION }\end{array}$ & 3.84 & 1.62 & 5.74 & .94 & 4.31 & 1.70 & 3.92 & 1.62 \\
\hline (28) violent overthrow & 5.57 & 1.53 & 5.59 & .91 & 5.34 & 1.29 & 4.44 & 1.72 \\
\hline $\begin{array}{l}\text { (07) turn in a circle } \\
\text { RICK }\end{array}$ & 3.68 & 1.80 & 4.97 & & 1.40 & 3.69 & 1.71 \\
\hline (20) stack of hay & 5.56 & 1.55 & 5.14 & 1.46 & 3.26 & 2.05 & 4.89 & 2.17 \\
\hline (05) name & 3.00 & 2.21 & 5.11 & 1.60 & 3.37 & 1.76 & 3.69 & 2.07 \\
\hline $\begin{array}{l}\text { (04) injure/jerk } \\
\text { RING }\end{array}$ & 2.60 & 1.62 & 6.23 & .93 & 1.97 & 1.11 & 3.14 & 1.84 \\
\hline (25) jewellery & 5.95 & 1.49 & 2.83 & 1.11 & 5.91 & 1.03 & 6.11 & 1.37 \\
\hline (10) circular form & 4.97 & 1.94 & 3.11 & 1.21 & 4.56 & 1.50 & 4.42 & 1.77 \\
\hline $\begin{array}{l}\text { (04) sound of bell } \\
\text { ROD }\end{array}$ & 5.81 & & 2.56 & .83 & 5.74 & 1.18 & 5.14 & 1.64 \\
\hline (18) fishing equipment & 6.11 & .98 & 3.39 & 1.01 & 4.83 & 1.52 & 5.61 & 1.66 \\
\hline (16) long thin solid object & 5.08 & 1.63 & 3.66 & 1.33 & 4.97 & 1.44 & 5.47 & 1.68 \\
\hline $\begin{array}{l}\text { (05) name } \\
\text { ROMANCE }\end{array}$ & 3.41 & 2.24 & 4.83 & 1.56 & 3.14 & 1.68 & 3.36 & 2.14 \\
\hline (36) love & 5.14 & 1.75 & 4.97 & .94 & 5.29 & 1.49 & 3.94 & 2.12 \\
\hline $\begin{array}{l}\text { (04) story/novel } \\
\text { ROOM }\end{array}$ & \multicolumn{7}{|c|}{ ROOM } & 1.80 \\
\hline (33) part of building & 5.84 & 1.44 & 2.23 & .64 & 6.46 & .84 & 5.81 & 1.45 \\
\hline $\begin{array}{l}\text { (07) space } \\
\text { RUBBER }\end{array}$ & 4.27 & 1.70 & 2.94 & 1.03 & 5.09 & 1.46 & 3.72 & 2.00 \\
\hline (17) eraser & 5.86 & 1.58 & 2.69 & .62 & 5.86 & 1.13 & 5.97 & 1.55 \\
\hline (16) material & 5.14 & 1.49 & 3.51 & 1.00 & 5.03 & 1.59 & 5.56 & 1.71 \\
\hline (07) plant & 5.43 & 1.79 & 4.69 & 1.12 & 3.89 & 1.70 & 5.06 & 1.96 \\
\hline
\end{tabular}




\begin{tabular}{|c|c|c|c|c|c|c|c|c|}
\hline \multirow[b]{2}{*}{ Word and Meaning } & \multicolumn{2}{|c|}{ Imagery } & \multicolumn{2}{|c|}{ Age of Acquisition } & \multicolumn{2}{|c|}{ Familiarity } & \multicolumn{2}{|c|}{ Concreteness } \\
\hline & Mean & SD & Mean & SD & Mean & SD & Mean & SD \\
\hline \multicolumn{9}{|l|}{ RULER } \\
\hline (32) measuring instrument & 6.22 & 1.04 & 2.91 & .55 & 6.14 & 1.07 & 6.11 & 1.17 \\
\hline $\begin{array}{l}\text { (08) one with power } \\
\text { SAMPLER }\end{array}$ & 4.54 & 1.80 & 3.86 & .99 & 4.11 & 1.45 & 3.69 & 1.68 \\
\hline (19) one who tests & 3.46 & 1.88 & 5.80 & 1.09 & 4.03 & 1.54 & 4.44 & 1.83 \\
\hline (12) embroidery & 3.83 & 2.27 & 5.80 & 1.21 & 2.63 & 2.02 & 3.94 & 2.20 \\
\hline (06) specimen & 4.11 & 1.57 & 5.69 & 1.05 & 3.46 & 1.68 & 4.64 & 1.70 \\
\hline \multicolumn{9}{|l|}{ SAVAGE } \\
\hline (25) uncivilised person & $\begin{array}{l}5.30 \\
5.00\end{array}$ & $\begin{array}{l}1.71 \\
1.66\end{array}$ & 4.06 & $\begin{array}{r}1.26 \\
94\end{array}$ & $\begin{array}{l}4.54 \\
4.66\end{array}$ & 1.70 & 5.17 & 1.85 \\
\hline \multicolumn{9}{|l|}{ SCENE } \\
\hline (22) setting/view & 4.89 & 1.49 & 4.39 & 1.04 & 4.88 & 1.53 & 4.67 & 1.62 \\
\hline (15) part of play & 4.97 & 1.77 & 4.67 & 1.11 & 4.91 & 1.44 & 4.94 & 1.55 \\
\hline \multicolumn{9}{|l|}{ SCOUT } \\
\hline (28) boy's organisation & 5.84 & 1.44 & 3.78 & .82 & 5.00 & 1.68 & 5.49 & 1.59 \\
\hline (11) to reconnoitre & 3.03 & 1.44 & 4.83 & 1.01 & 3.26 & 1.48 & 3.25 & 1.62 \\
\hline \multicolumn{9}{|l|}{ SCULL } \\
\hline (24) rowing & 3.16 & 2.11 & 6.33 & .78 & 2.54 & 1.59 & 4.08 & 1.53 \\
\hline \multirow{2}{*}{\multicolumn{9}{|c|}{ SEAL }} \\
\hline & & & & & & & & \\
\hline $\begin{array}{l}\text { (29) animal } \\
\text { (07) enclose }\end{array}$ & $\begin{array}{l}6.41 \\
4.03\end{array}$ & 1.10 & 3.22 & 1.08 & 5.03 & 1.40 & 6.50 & 1.04 \\
\hline \multicolumn{9}{|l|}{$\begin{array}{l}\text { (0/) enclose } \\
\text { SEASON }\end{array}$} \\
\hline (34) time & 5.23 & 1.57 & 3.57 & 1.20 & 5.29 & 1.36 & 4.58 & 1.96 \\
\hline (04) flavour & 4.64 & 1.69 & 5.14 & 1.08 & 4.43 & 1.59 & 4.28 & 1.52 \\
\hline SECOND & & & & & & & & \\
\hline (27) measure of time & 4.24 & 1.94 & 3.17 & .77 & 5.94 & 1.08 & 3.81 & 2.28 \\
\hline (12) next after first & 3.62 & 2.17 & 2.53 & .83 & 6.23 & .96 & 4.22 & 2.37 \\
\hline SELECTION & & & & & & & & \\
\hline (35) choice & 4.27 & 2.10 & 3.97 & 1.04 & 5.57 & 1.23 & 4.22 & 1.81 \\
\hline (05) collection affording choice & 3.89 & 1.91 & 4.36 & 1.06 & 5.20 & 1.37 & 4.00 & 2.06 \\
\hline SENSE & & & & & & & & \\
\hline (17) stimuli receptor & 3.24 & 1.85 & 5.89 & 1.20 & 4.68 & 1.34 & 3.50 & 1.83 \\
\hline (13) wisdom & 3.49 & 1.95 & 4.39 & 1.21 & 5.34 & 1.07 & 3.42 & 2.11 \\
\hline (10) feeling/hunch & 3.89 & 1.93 & 4.94 & 1.15 & 4.54 & 1.34 & 3.58 & 1.98 \\
\hline SERIES & & & & & & & & \\
\hline (27) sequence of stories & 4.11 & 1.67 & 4.81 & 1.10 & 4.71 & 1.49 & 4.29 & 1.58 \\
\hline (13) related collection & 3.19 & 1.89 & 4.97 & 1.01 & 4.63 & 1.24 & 3.56 & 1.83 \\
\hline SER VICE & & & & & & & & \\
\hline (26) work done for another & 3.27 & 1.67 & 5.00 & 1.01 & 4.80 & 1.35 & 3.78 & 1.67 \\
\hline (06) religious gathering & 5.17 & 1.52 & 3.56 & 1.14 & 4.54 & 1.75 & 4.75 & 2.02 \\
\hline (05) to maintain & 2.97 & 1.69 & 5.14 & 1.00 & 4.09 & 1.42 & 3.65 & 1.73 \\
\hline SETTLEMENT & & & & & & & & \\
\hline (28) colony & 5.14 & 1.65 & 5.03 & 1.08 & 4.43 & 1.52 & 5.03 & 1.46 \\
\hline (08) agreement & 3.92 & 1.85 & 5.29 & 1.00 & 4.57 & 1.32 & 3.67 & 1.68 \\
\hline (04) amount of money & 3.62 & 1.95 & 6.19 & .81 & 3.54 & 1.32 & 3.44 & 1.85 \\
\hline SEX & & & & & & & & \\
\hline (23) classification & 4.36 & 1.65 & 4.69 & 1.31 & 5.06 & 1.45 & 3.86 & 1.95 \\
\hline (17) intercourse & 6.31 & 1.17 & 5.42 & 1.04 & 5.74 & 1.15 & 6.06 & 1.17 \\
\hline SHAKER & & & & & & & & \\
\hline (27) mixer & 4.58 & 1.92 & 4.92 & 1.14 & 3.94 & 1.45 & 5.08 & 1.42 \\
\hline (06) noise maker & 3.68 & 1.96 & 3.69 & 1.56 & 3.09 & 1.57 & 4.36 & 1.86 \\
\hline SHAME & & & & & & & & \\
\hline (31) feeling of disgrace & 4.62 & 1.91 & 3.83 & 1.14 & 5.49 & 1.44 & 4.11 & 2.08 \\
\hline (09) pity & 3.03 & 1.68 & 3.92 & 1.19 & 5.09 & 1.72 & 3.83 & 2.02 \\
\hline SHARPNESS & & & & & & & & \\
\hline (34) having fine point & 4.94 & 1.72 & 3.78 & 1.38 & 5.17 & 1.59 & 4.22 & 1.80 \\
\hline (04) quick-witted & 3.41 & 1.95 & 5.11 & 1.13 & 4.14 & 1.50 & 3.03 & 1.72 \\
\hline SHED & & & & & & & & \\
\hline (35) hut & 5.92 & 1.24 & 2.78 & .89 & 5.51 & 1.13 & 5.72 & 1.68 \\
\hline (05) get rid of & 3.75 & 1.66 & 4.81 & 1.02 & 3.66 & 1.37 & 3.69 & 1.71 \\
\hline SHEET & & & & & & & & \\
\hline (31) bed covering & 6.11 & 1.31 & 2.53 & .93 & 5.97 & .94 & 6.31 & .91 \\
\hline (07) piece of paper & 5.54 & 1.48 & 2.94 & .72 & 5.60 & 1.15 & 5.33 & 1.78 \\
\hline SHIELD & & & & & & & & \\
\hline (22) protection & 5.11 & 1.52 & 3.53 & 1.01 & 4.43 & 1.63 & 5.17 & 1.54 \\
\hline (17) armour & 5.46 & 1.43 & 3.61 & 1.16 & 4.03 & 1.46 & 5.67 & 1.43 \\
\hline
\end{tabular}




\begin{tabular}{|c|c|c|c|c|c|c|c|c|}
\hline \multirow[b]{2}{*}{ Word and Meaning } & \multicolumn{2}{|c|}{ Imagery } & \multicolumn{2}{|c|}{ Age of Acquisition } & \multicolumn{2}{|c|}{ Familiarity } & \multicolumn{2}{|c|}{ Concreteness } \\
\hline & Mean & SD & Mean & SD & Mean & SD & Mean & SD \\
\hline \multicolumn{9}{|l|}{ SHOWER } \\
\hline (23) method of cleaning & 5.97 & 1.20 & 4.11 & 1.33 & 5.86 & 1.10 & 5.92 & 1.23 \\
\hline (16) rain & 6.28 & .84 & 2.97 & .93 & 5.17 & 1.23 & 5.03 & 1.85 \\
\hline \multicolumn{9}{|l|}{ SHUTTER } \\
\hline (29) window covering & 5.72 & 1.37 & 4.06 & 1.03 & 4.63 & 1.66 & 6.00 & 1.29 \\
\hline \multicolumn{8}{|l|}{ SINK } & 1.42 \\
\hline (26) receptacle for washing & 6.11 & 1.07 & 2.61 & .95 & 6.29 & .97 & 6.39 & .98 \\
\hline (10) become submerged & 4.69 & 1.47 & 3.42 & 1.01 & 4.54 & 1.38 & 3.72 & 1.59 \\
\hline \multicolumn{9}{|l|}{ SIREN } \\
\hline (34) loud warning noise & 5.61 & 1.57 & 3.92 & 1.23 & 5.26 & 1.40 & 5.61 & 1.44 \\
\hline (05) horn & 5.65 & 1.56 & 3.81 & 1.15 & 4.97 & 1.61 & 6.00 & 1.03 \\
\hline \multicolumn{9}{|l|}{ SKIRT } \\
\hline (36) clothing & 6.19 & 1.20 & 2.64 & 1.00 & 6.00 & 1.22 & 6.22 & 1.25 \\
\hline \multirow{2}{*}{\multicolumn{9}{|c|}{ SLAVE }} \\
\hline & & & & & & & & \\
\hline (36) unpaid servant & 5.72 & 1.45 & 3.78 & 1.13 & 5.00 & 1.55 & 5.67 & 1.56 \\
\hline (04) to work hard & 4.19 & 1.92 & 5.11 & 1.07 & 4.20 & 1.33 & 3.94 & 1.62 \\
\hline \multicolumn{9}{|l|}{ SMASH } \\
\hline (32) break violently & 5.92 & 1.24 & 3.06 & .75 & 5.63 & 1.38 & 5.20 & 1.45 \\
\hline (04) hit hard in sport & 4.61 & 1.88 & 4.83 & 1.09 & 4.11 & 1.70 & 4.00 & 1.70 \\
\hline (04) brand of dried potato & 4.86 & 1.92 & 6.06 & 1.15 & 3.53 & 1.61 & 4.57 & 2.25 \\
\hline SMOKE & & & & & & & & \\
\hline (25) cloudy gas from fire & 6.14 & 1.12 & 2.71 & 1.08 & 5.77 & 1.17 & 5.47 & 1.61 \\
\hline (15) inhale from cigarette & 5.42 & 1.66 & 3.83 & 1.16 & 5.57 & 1.50 & 5.58 & 1.71 \\
\hline SNORT & & & & & & & & \\
\hline (26) derisory grunt & 5.03 & 1.84 & 3.91 & 1.08 & 4.54 & 1.56 & 4.61 & 1.83 \\
\hline (13) noise of pig/horse, etc. & 5.50 & 1.56 & 2.69 & 1.10 & 4.89 & 1.69 & 5.22 & 1.64 \\
\hline SOIL & & 163 & & & & & & \\
\hline $\begin{array}{l}\text { (25) earth } \\
\text { (05) stain }\end{array}$ & $\begin{array}{l}5.92 \\
4.27\end{array}$ & $\begin{array}{l}1.63 \\
1.80\end{array}$ & $\begin{array}{l}3.29 \\
4.94\end{array}$ & 1.08 & 5.31 & 1.43 & 6.00 & 1.27 \\
\hline $\begin{array}{l}\text { (05) stain } \\
\text { SOLUTION }\end{array}$ & 4.27 & 1.80 & 4.94 & 1.22 & 3.74 & 1.38 & 3.78 & 1.65 \\
\hline (27) answer & 4.08 & 2.25 & 4.50 & 1.12 & 5.83 & 1.06 & 4.00 & 2.07 \\
\hline (13) solid dissolved in liquid & 4.32 & 1.73 & 6.00 & .89 & 5.11 & 1.35 & 4.56 & 1.82 \\
\hline SPACE & & & & & & & & \\
\hline (26) expanse of universe & 4.68 & 2.03 & 4.19 & 1.17 & 5.43 & 1.32 & 4.17 & 2.21 \\
\hline (14) open area & 4.97 & 1.76 & 3.11 & .99 & 5.34 & 1.35 & 3.86 & 1.86 \\
\hline SPAN & & & & & & & & \\
\hline (27) extent & 3.19 & 1.90 & 5.23 & 1.17 & 4.11 & 1.43 & 3.22 & 1.75 \\
\hline (11) stretch of hand & 5.30 & 1.74 & 4.71 & 1.30 & 3.80 & 1.45 & 4.75 & 1.79 \\
\hline SPEECH & & & & & & & & \\
\hline (20) spoken language & 4.62 & 1.99 & 3.40 & .87 & 6.06 & 1.19 & 5.33 & 1.83 \\
\hline (20) oration & 5.11 & 1.70 & 3.72 & 1.28 & 5.03 & 1.46 & 5.14 & 1.64 \\
\hline SPELL & & & & & & & & \\
\hline (21) words and letters & 3.89 & 1.91 & 2.97 & .73 & 5.51 & 1.48 & 4.00 & 2.20 \\
\hline (15) magic & 4.83 & 1.44 & 2.78 & 1.06 & 3.91 & 1.61 & 3.36 & 2.00 \\
\hline SPIRIT & & & & & & & & \\
\hline (25) drink & 5.61 & 1.51 & 5.29 & 1.11 & 5.29 & 1.36 & 5.42 & 1.77 \\
\hline (09) ghost & 5.36 & 1.60 & 3.92 & 1.12 & 4.20 & 1.60 & 4.03 & 2.29 \\
\hline SPOKE & & & & & & & & \\
\hline (32) metal rod & 5.17 & 1.46 & 4.03 & 1.19 & 4.49 & 1.75 & 5.08 & 2.07 \\
\hline (08) did speak & 3.16 & 2.25 & 3.11 & 1.01 & 5.51 & 1.59 & 4.17 & 1.99 \\
\hline SPOT & & & & & & & & \\
\hline (26) mark & 5.44 & 1.50 & 2.89 & .97 & 5.17 & 1.34 & 5.22 & 1.47 \\
\hline (05) pimple & 5.97 & 1.12 & 4.33 & 1.47 & 5.43 & 1.32 & 5.81 & 1.31 \\
\hline (05) place & 3.76 & 1.81 & 3.66 & .83 & 4.40 & 1.50 & 4.25 & 1.53 \\
\hline (04) to notice & 3.72 & 1.50 & 3.69 & 1.05 & 4.66 & 1.45 & 3.61 & 1.64 \\
\hline SPURT & & & & & & & & \\
\hline (23) extra energy & 4.57 & 1.84 & 4.57 & 1.08 & 4.40 & 1.36 & 3.92 & 1.62 \\
\hline (15) sudden flow of liquid & 5.53 & 1.52 & 4.11 & 1.22 & 4.91 & 1.36 & 5.00 & 1.56 \\
\hline STABLE & & & & & & & & \\
\hline (29) shelter for horses & 6.33 & 1.13 & 2.81 & 1.02 & 5.21 & 1.47 & 6.36 & .98 \\
\hline $\begin{array}{l}\text { (11) balanced } \\
\text { STAFF }\end{array}$ & 3.43 & 1.78 & 5.42 & 1.12 & 4.69 & 1.35 & 3.17 & 1.56 \\
\hline (35) employees & 5.28 & 1.57 & 4.60 & 1.05 & 5.74 & 1.16 & 5.86 & 1.16 \\
\hline (04) wooden stick & 5.38 & 1.46 & 4.25 & 1.01 & 3.63 & 1.66 & 5.17 & 1.97 \\
\hline
\end{tabular}




\begin{tabular}{|c|c|c|c|c|c|c|c|c|}
\hline \multirow[b]{2}{*}{ Word and Meaning } & \multicolumn{2}{|c|}{ Imagery } & \multicolumn{2}{|c|}{ Age of Acquisition } & \multicolumn{2}{|c|}{ Familiarity } & \multicolumn{2}{|c|}{ Concreteness } \\
\hline & Mean & SD & Mean & SD & Mean & SD & Mean & SD \\
\hline \multicolumn{9}{|l|}{ STALK } \\
\hline (22) stem of plant & 5.78 & 1.32 & 3.77 & 1.20 & 4.91 & 1.46 & 5.67 & 1.41 \\
\hline (17) hunt & 5.22 & 1.25 & 4.39 & .83 & 4.03 & 1.63 & 4.39 & 1.78 \\
\hline \multicolumn{9}{|l|}{ STARCH } \\
\hline (19) material for stiffening cloth & 4.60 & 1.88 & 5.17 & 1.38 & 4.49 & 1.65 & 5.33 & 1.84 \\
\hline (12) contained in bread/potatoes & 3.83 & 2.10 & 5.51 & .97 & 5.29 & 1.52 & 4.81 & 1.93 \\
\hline (09) chemical & 3.65 & 2.07 & 5.97 & .83 & 4.20 & 1.67 & 4.83 & 1.86 \\
\hline \multicolumn{9}{|l|}{ STATEMENT } \\
\hline (33) expression in words & 3.57 & 1.64 & 4.97 & .96 & 5.11 & 1.37 & 3.97 & 1.71 \\
\hline \\
\hline (31) stopping place for trains, etc. & 6.28 & 1.07 & 2.72 & .73 & 6.31 & .92 & 6.44 & .83 \\
\hline (05) employed position & 2.68 & 1.47 & 5.43 & .90 & 2.91 & 1.23 & 3.00 & 1.60 \\
\hline (04) base for police/firemen, etc. & 5.16 & 1.69 & 2.86 & .86 & 5.34 & 1.39 & 4.78 & 1.84 \\
\hline \multicolumn{9}{|l|}{ STEP } \\
\hline (20) to walk & $\begin{array}{l}4.68 \\
5.89\end{array}$ & $\begin{array}{l}1.68 \\
1.39\end{array}$ & $\begin{array}{l}2.83 \\
2.53\end{array}$ & $\begin{array}{r}1.16 \\
.83\end{array}$ & $\begin{array}{l}4.91 \\
5.43\end{array}$ & $\begin{array}{l}1.27 \\
1.42\end{array}$ & $\begin{array}{l}4.61 \\
5.86\end{array}$ & $\begin{array}{l}1.53 \\
1.53\end{array}$ \\
\hline $\begin{array}{l}\text { (18) stair } \\
\text { STORK }\end{array}$ & \multicolumn{6}{|c|}{ STORK } & & \\
\hline (36) bird & 6.08 & 1.17 & 3.89 & 1.24 & 4.34 & 1.97 & 5.67 & 1.62 \\
\hline (04) margarine & 5.72 & 1.59 & 5.61 & 1.23 & 4.54 & 1.56 & 5.22 & 2.06 \\
\hline \multicolumn{9}{|l|}{ STRAIN } \\
\hline (23) tension & 4.33 & 1.75 & 5.17 & 1.09 & 5.26 & 1.16 & 3.94 & 1.96 \\
\hline (10) attempt & 3.11 & 1.93 & 4.97 & 1.04 & 3.14 & 1.57 & 3.14 & 1.36 \\
\hline (05) sieve & 4.61 & 1.62 & 4.39 & 1.25 & 4.26 & 1.71 & 4.28 & 1.94 \\
\hline \multicolumn{9}{|l|}{ STUMP } \\
\hline (24) remains of tree & 5.89 & 1.25 & 3.69 & 1.12 & 4.57 & 1.59 & 5.78 & 1.60 \\
\hline (09) remains of limb & 4.74 & 1.87 & 4.94 & 1.33 & 3.56 & 1.52 & 5.06 & 1.84 \\
\hline (04) end & 4.19 & 1.71 & 4.37 & 1.17 & 3.94 & 1.55 & 4.44 & 1.59 \\
\hline \multicolumn{9}{|l|}{ SUBJECT } \\
\hline (20) topic & 3.38 & 1.98 & 4.49 & 1.13 & 5.74 & 1.10 & 3.89 & 2.12 \\
\hline (17) person in experiment & 5.11 & 1.50 & 6.53 & .65 & 5.23 & 1.40 & 5.50 & 1.69 \\
\hline $\begin{array}{l}\text { SUM } \\
\text { (23) calculation }\end{array}$ & 5.28 & 1.63 & 2.92 & 72 & & & & \\
\hline $\begin{array}{l}\text { (23) calculation } \\
\text { (12) total }\end{array}$ & $\begin{array}{l}3.20 \\
3.70\end{array}$ & 2.03 & 3.61 & 1.09 & $\begin{array}{l}3.09 \\
5.74\end{array}$ & $\begin{array}{l}1.26 \\
1.13\end{array}$ & $\begin{array}{l}4.94 \\
4.14\end{array}$ & $\begin{array}{l}1.83 \\
1.95\end{array}$ \\
\hline $\begin{array}{l}\text { (12) total } \\
\text { (04) money }\end{array}$ & 4.53 & 1.77 & 4.06 & .94 & 4.74 & 1.18 & $\begin{array}{l}4.14 \\
4.08\end{array}$ & $\begin{array}{l}1.90 \\
1.52\end{array}$ \\
\hline SURFACE & & & & & & & & \\
\hline (35) exterior of a body & 4.14 & 1.69 & 4.36 & 1.38 & 5.23 & 1.49 & 4.44 & 1.85 \\
\hline (05) top of liquid & 4.76 & 1.78 & 4.28 & 1.24 & 4.23 & 1.59 & 4.67 & 1.86 \\
\hline SUSPECT & & & & & & & & \\
\hline (20) potential criminal & 4.51 & 1.84 & 5.25 & .98 & 4.60 & 1.22 & 4.56 & 1.76 \\
\hline (10) dubious/not trustworthy & 3.92 & 1.77 & 5.22 & .92 & 4.46 & 1.36 & 3.42 & 1.89 \\
\hline (10) have a notion that & 3.39 & 2.00 & 4.67 & 1.05 & 5.06 & 1.39 & 3.60 & 1.86 \\
\hline $\begin{array}{l}\text { SWALLOW } \\
\text { (29) bird }\end{array}$ & 6.36 & 1.06 & 3.57 & 1.05 & 4.80 & 1.45 & 5.53 & 1.99 \\
\hline (11) food intake & 5.19 & 1.51 & 2.72 & 1.10 & 5.71 & 1.25 & 5.17 & 1.61 \\
\hline SWEEP & & & & & & & & \\
\hline (29) use a brush & 5.62 & 1.34 & 3.00 & .94 & 5.46 & 1.44 & 4.92 & 1.53 \\
\hline $\begin{array}{l}\text { (08) person who cleans chimneys } \\
\text { TAP }\end{array}$ & 5.17 & 1.68 & 3.39 & 1.06 & 3.94 & 1.97 & 5.67 & 1.51 \\
\hline $\begin{array}{l}\text { TAP } \\
\text { (22) faucet }\end{array}$ & 4.32 & 2.35 & 3.31 & 1.70 & 4.03 & 2.38 & 5.03 & 1.92 \\
\hline (16) knock lightly & 4.83 & 1.65 & 3.14 & .90 & 4.66 & 1.17 & 4.53 & 1.83 \\
\hline TAPE & & & & & & & & \\
\hline (27) recording of sounds & 5.14 & 1.51 & 4.77 & 1.15 & 5.83 & 1.21 & 5.56 & 1.38 \\
\hline (11) ribbon & 5.38 & 1.63 & 3.71 & 1.19 & 4.29 & 1.58 & 5.42 & 1.69 \\
\hline TEAR & & & & & & & & \\
\hline (21) rip & 5.38 & 1.42 & 2.97 & .97 & 5.66 & 1.31 & 5.25 & 1.32 \\
\hline (19) liquid from eye & 5.84 & 1.37 & 2.40 & .90 & 5.60 & 1.25 & 5.78 & 1.72 \\
\hline TERM & & & & & & & & \\
\hline (24) part of academic year & 4.92 & 1.99 & 3.86 & 1.11 & 6.14 & .93 & 4.44 & 1.92 \\
\hline $\begin{array}{l}\text { (13) period of time } \\
\text { TIN }\end{array}$ & 3.36 & 2.02 & 4.31 & 1.24 & 5.20 & 1.21 & 3.44 & 1.88 \\
\hline $\begin{array}{l}\text { TIN } \\
\text { (24) metal }\end{array}$ & 4.69 & 1.97 & 4.22 & 1.06 & 5.31 & 1.37 & 5.25 & 1.82 \\
\hline (16) container & 5.97 & 1.28 & 2.66 & 1.01 & 5.57 & 1.34 & 5.94 & 1.45 \\
\hline TITLE & & & & & & & & \\
\hline (28) heading & 5.03 & 1.78 & 3.81 & .88 & 5.89 & 1.06 & 4.86 & 1.78 \\
\hline (12) denoting rank & 4.03 & 1.79 & 4.78 & 1.34 & 4.34 & 1.53 & 3.63 & 2.02 \\
\hline
\end{tabular}




\begin{tabular}{|c|c|c|c|c|c|c|c|c|}
\hline \multirow[b]{2}{*}{ Word and Meaning } & \multicolumn{2}{|c|}{ Imagery } & \multicolumn{2}{|c|}{ Age of Acquisition } & \multicolumn{2}{|c|}{ Familiarity } & \multicolumn{2}{|c|}{ Concreteness } \\
\hline & Mean & SD & Mean & SD & Mean & SD & Mean & SD \\
\hline \multicolumn{9}{|l|}{ TOKEN } \\
\hline (19) symbol & 3.49 & 1.84 & 5.53 & 1.24 & 4.00 & 1.43 & 3.78 & 1.78 \\
\hline (12) gift & 4.43 & 1.67 & 5.03 & 1.26 & 4.29 & 1.70 & 4.92 & 1.52 \\
\hline (07) coin & 4.31 & 1.94 & 5.20 & 1.33 & 3.06 & 1.35 & 4.92 & 1.67 \\
\hline \multicolumn{9}{|l|}{ TOPIC } \\
\hline (29) subject type & 3.49 & 2.02 & 4.92 & 1.16 & 5.63 & 1.02 & 3.94 & 1.83 \\
\hline (10) chocolate & 5.36 & 1.70 & 5.36 & 1.23 & 3.91 & 1.76 & 4.78 & 2.32 \\
\hline \multicolumn{9}{|l|}{ TRACK } \\
\hline (14) road & 5.75 & 1.16 & 3.64 & 1.03 & 4.49 & 1.34 & 5.22 & 1.27 \\
\hline (09) railway & 5.92 & 1.30 & 3.11 & 1.13 & 5.09 & 1.38 & 5.97 & 1.59 \\
\hline (06) trail left by animal & 4.68 & 1.54 & 3.86 & .92 & 3.77 & 1.20 & 4.89 & 1.49 \\
\hline (05) to follow & 4.67 & 1.47 & 4.50 & 1.07 & 3.77 & 1.22 & 4.25 & 1.46 \\
\hline \multicolumn{9}{|l|}{ TRANSFER } \\
\hline (29) change position & 3.49 & 1.87 & 5.25 & 1.04 & 5.37 & 1.33 & 3.61 & 1.86 \\
\hline (10) swap/hand over & 4.33 & 1.94 & 4.61 & 1.11 & 5.00 & 1.41 & 4.22 & 1.64 \\
\hline \multicolumn{9}{|l|}{ TRANSFORMER } \\
\hline (34) for altering voltage & 3.65 & 2.28 & 6.53 & .73 & 3.97 & 1.96 & 5.28 & 1.95 \\
\hline $\begin{array}{l}\text { (04) object/person causing change } \\
\text { TRIAL }\end{array}$ & 2.03 & 1.40 & 5.94 & 1.00 & 2.69 & 1.33 & 3.47 & 1.77 \\
\hline (29) legal proceeding & 5.14 & 1.57 & 4.94 & .88 & 5.09 & 1.42 & 4.40 & 1.81 \\
\hline (07) test & 4.16 & 1.55 & 4.89 & 1.10 & 4.46 & 1.20 & 4.03 & 1.72 \\
\hline \multicolumn{9}{|l|}{ TRICK } \\
\hline (20) performed skill & 4.08 & 1.87 & 3.36 & .98 & 4.69 & 1.60 & 4.19 & 1.68 \\
\hline (12) to cheat & 3.92 & 1.89 & 3.47 & .87 & 4.46 & 1.38 & 4.31 & 1.85 \\
\hline (08) practical joke & 4.41 & 1.53 & 3.42 & 1.01 & 5.03 & 1.48 & 4.50 & 1.46 \\
\hline TRIM & & & & & & & & \\
\hline (28) cut slightly & 5.05 & 1.59 & 3.64 & 1.11 & 5.29 & 1.30 & 4.81 & 1.66 \\
\hline (06) finishing touches & 4.19 & 1.76 & 5.11 & 1.10 & 4.40 & 1.63 & 3.78 & 1.55 \\
\hline$(06)$ neat/thin & 3.95 & 1.75 & 5.14 & 1.08 & 3.68 & 1.66 & 3.17 & 1.46 \\
\hline TRIP & & & & & & & & \\
\hline (21) journey & 4.95 & 1.71 & 3.42 & 1.01 & 5.49 & 1.18 & 4.64 & 1.90 \\
\hline (16) fall & 5.41 & 1.37 & 2.69 & .82 & 5.20 & 1.28 & 4.72 & 1.43 \\
\hline TRIUMPH & & & & & & & & \\
\hline (20) success & 4.31 & 1.68 & 4.81 & .97 & 4.71 & 1.39 & 3.92 & 2.34 \\
\hline (18) make of car & 5.14 & 1.79 & 4.66 & 1.24 & 4.37 & 1.49 & 5.11 & 2.01 \\
\hline TRUNK & & & & & & & & \\
\hline (26) box/container & 5.94 & 1.27 & 3.92 & 1.19 & 4.69 & 1.49 & 6.17 & 1.21 \\
\hline (06) stem of tree & 6.08 & 1.02 & 2.92 & 1.01 & 5.49 & 1.32 & 6.06 & 1.49 \\
\hline (05) elephant's proboscis & 6.22 & 1.02 & 2.67 & .94 & 4.89 & 1.65 & 5.50 & 2.02 \\
\hline TUCK & & & & & & & & \\
\hline (21) fold in cloth & 4.38 & 1.81 & 4.19 & 1.58 & 4.11 & 1.69 & 4.78 & 1.80 \\
\hline (13) food/eat & 4.05 & 1.83 & 4.53 & 1.30 & 2.74 & 1.56 & 4.47 & 1.97 \\
\hline (06) put between & 3.89 & 1.94 & 3.53 & 1.40 & 4.43 & 1.42 & 3.56 & 1.77 \\
\hline TURN & & & & & & & & \\
\hline (18) rotate & 4.75 & 1.96 & 2.61 & 1.01 & 6.00 & 1.17 & 4.72 & 1.63 \\
\hline (14) change direction & 4.39 & 1.74 & 2.60 & .90 & 5.89 & .92 & 4.94 & 1.65 \\
\hline (04) recurring opportunity & 2.92 & 1.48 & 3.94 & 1.47 & 4.11 & 1.67 & 3.33 & 1.60 \\
\hline TURNER & & & & & & & & \\
\hline (09) work on lathe & 2.84 & 1.97 & 6.69 & .52 & 2.60 & 1.79 & 4.11 & 2.08 \\
\hline (09) one who turns & 2.89 & 1.81 & 4.64 & 1.34 & 2.83 & 1.30 & 4.31 & 1.90 \\
\hline (08) the artist & 4.00 & 2.36 & 6.33 & .67 & 3.38 & 2.07 & 4.47 & 2.23 \\
\hline (07) name & 2.73 & 2.39 & 4.94 & 1.47 & 2.37 & 1.22 & 3.58 & 2.47 \\
\hline TYPE & & & & & & & & \\
\hline (28) kind of & 2.78 & 1.96 & 3.78 & .92 & 6.29 & .81 & 3.61 & 1.92 \\
\hline (12) print & 5.47 & 1.69 & 4.53 & .99 & 4.60 & 1.52 & 4.72 & 1.56 \\
\hline UNDERTAKING & & & & & & & & \\
\hline (20) responsibility & 3.11 & 1.89 & 5.61 & .86 & 4.38 & 1.53 & 3.53 & 1.83 \\
\hline (14) assurance & 2.50 & 1.61 & 6.19 & .78 & 3.40 & 1.44 & 2.75 & 1.42 \\
\hline (05) funeral arrangements & 3.73 & 2.11 & 5.56 & 1.12 & 3.06 & 1.33 & 3.47 & 2.03 \\
\hline UNDOING & & & & & & & & 2.05 \\
\hline (18) ruin & 3.05 & 1.83 & 5.47 & 1.07 & 3.82 & 1.79 & 3.33 & 1.72 \\
\hline (18) unfastening & 4.87 & 1.85 & 3.19 & 1.27 & 5.29 & 1.32 & 4.75 & 1.53 \\
\hline UNIFORM & & & & & & & & \\
\hline (30) set of clothes & 6.00 & 1.23 & 3.09 & .94 & 5.40 & 1.53 & 6.39 & .86 \\
\hline (10) sameness & 3.49 & 1.64 & 5.19 & 1.24 & 4.26 & 1.61 & 2.89 & 1.58 \\
\hline UPSET & & & & & & & & \\
\hline (28) distress & 4.56 & 1.80 & 3.50 & 1.09 & 5.57 & 1.27 & 4.33 & 1.99 \\
\hline
\end{tabular}




\begin{tabular}{|c|c|c|c|c|c|c|c|c|}
\hline \multirow[b]{2}{*}{ Word and Meaning } & \multicolumn{2}{|c|}{ Imagery } & \multicolumn{2}{|c|}{ Age of Acquisition } & \multicolumn{2}{|c|}{ Familiarity } & \multicolumn{2}{|c|}{ Concreteness } \\
\hline & Mean & SD & Mean & SD & Mean & SD & Mean & SD \\
\hline $\begin{array}{l}\text { (11) overturn } \\
\text { URCHIN }\end{array}$ & 4.32 & 1.74 & 3.89 & 1.22 & 3.89 & 1.60 & 3.92 & 1.52 \\
\hline (31) mischievous/neglected child & 5.31 & 1.63 & 5.17 & 1.14 & 4.11 & 1.91 & 5.25 & 1.75 \\
\hline $\begin{array}{l}\text { (08) sea creature } \\
\text { VENTURE }\end{array}$ & 4.95 & 1.58 & 4.17 & 1.48 & 3.71 & 1.78 & 5.44 & 1.83 \\
\hline (31) undertaking of risk & 3.24 & 1.81 & 5.47 & .90 & 4.29 & 1.34 & 3.64 & 1.72 \\
\hline $\begin{array}{l}\text { (07) journey } \\
\text { VESSEL }\end{array}$ & 4.08 & 1.53 & 5.26 & 1.16 & 4.03 & 1.32 & 3.97 & 1.61 \\
\hline (28) container & 4.68 & 1.69 & 4.75 & 1.26 & 4.41 & 1.57 & 5.19 & 1.76 \\
\hline $\begin{array}{l}\text { (12) ship } \\
\text { VICE }\end{array}$ & 5.62 & 1.40 & 4.50 & 1.04 & 4.11 & 1.70 & 5.89 & 1.37 \\
\hline (18) bad habit & 4.65 & 1.66 & 5.31 & 1.10 & 4.40 & 1.84 & 4.03 & 1.71 \\
\hline (12) tool for gripping & 5.19 & 1.86 & 5.06 & 1.17 & 4.03 & 1.70 & 5.03 & 1.99 \\
\hline $\begin{array}{l}\text { (07) evil } \\
\text { VISION }\end{array}$ & 3.92 & 1.70 & 5.40 & 1.13 & 4.17 & 1.81 & 3.33 & 1.92 \\
\hline (35) ability to see & 4.30 & 1.78 & 4.22 & .89 & 5.54 & 1.36 & 4.78 & 1.89 \\
\hline $\begin{array}{l}\text { (05) premonition } \\
\text { VOLUME }\end{array}$ & 3.51 & 1.80 & 5.39 & 1.09 & 3.89 & 1.64 & 3.03 & 1.72 \\
\hline (21) amount/capacity & 3.97 & 1.98 & 4.80 & 1.14 & 5.86 & .83 & 3.78 & 1.87 \\
\hline $\begin{array}{l}(16) \text { book } \\
\text { WASTE }\end{array}$ & 4.97 & 1.59 & 4.69 & .94 & 5.00 & 1.82 & 5.56 & 1.52 \\
\hline (24) garbage & 5.46 & 1.50 & 3.94 & .97 & 4.77 & 1.53 & 5.50 & 1.36 \\
\hline $\begin{array}{l}\text { (16) expend uselessly } \\
\text { WICK }\end{array}$ & 3.64 & 1.77 & 3.47 & 1.19 & 4.86 & 1.25 & 3.81 & 1.81 \\
\hline (33) string in candle & 5.81 & 1.45 & 4.28 & 1.37 & 5.03 & 1.67 & 4.69 & 1.76 \\
\hline $\begin{array}{l}\text { (07) town in Scotland } \\
\text { WONDER }\end{array}$ & 3.89 & 2.30 & 5.31 & 1.24 & 3.83 & 2.02 & 4.92 & 2.01 \\
\hline (16) puzzled state & 3.97 & 1.70 & 3.78 & 1.25 & 5.09 & 1.56 & 3.81 & 2.18 \\
\hline (14) to think & 3.54 & 2.11 & 3.80 & .98 & 5.57 & 1.42 & 3.50 & 2.29 \\
\hline $\begin{array}{l}\text { (07) a marvel } \\
\text { WOOD }\end{array}$ & 3.81 & 1.66 & 4.17 & 1.32 & 4.51 & 1.30 & 3.44 & 2.01 \\
\hline (27) material from tree & 6.19 & 1.49 & 2.33 & .67 & 6.14 & 1.15 & 6.11 & 1.37 \\
\hline $\begin{array}{l}\text { (13) collection of trees } \\
\text { WRECK }\end{array}$ & 6.36 & .79 & 2.58 & .83 & 5.26 & 1.34 & 6.06 & 1.55 \\
\hline (35) ruined ship/object & 5.97 & 1.32 & 3.36 & 1.03 & 4.97 & 1.59 & 5.94 & 1.56 \\
\hline $\begin{array}{l}\text { (05) to destroy } \\
\text { YARD }\end{array}$ & 5.03 & 1.55 & 3.42 & .80 & 5.17 & 1.34 & 4.83 & 1.59 \\
\hline $\begin{array}{l}\text { (28) unit of measurement } \\
\text { (09) area for working }\end{array}$ & $\begin{array}{l}5.22 \\
4.72\end{array}$ & $\begin{array}{l}1.77 \\
1.76\end{array}$ & $\begin{array}{l}3.25 \\
4.39\end{array}$ & $\begin{array}{r}.92 \\
1.21\end{array}$ & $\begin{array}{l}5.43 \\
4.14\end{array}$ & $\begin{array}{l}1.34 \\
1.44\end{array}$ & $\begin{array}{l}4.56 \\
5.14\end{array}$ & $\begin{array}{l}1.99 \\
1.62\end{array}$ \\
\hline
\end{tabular}

Note-In parentheses beside each meaning is the number of subjects (out of 40) who gave that meaning when asked to give the first meaning they thought of for the word concerned. Alongside each meaning are average scores for that meaning on imagery, age of acquisition, familiarity, and concreteness. Standard deviations (SDs) are given for each at tribute for each meaning. 